\title{
Binary Mean Field Stochastic Games: Stationary Equilibria and Comparative Statics*
}

\author{
Minyi Huang and Yan Ma
}

\begin{abstract}
This paper considers mean field games in a multi-agent Markov decision process (MDP) framework. Each player has a continuum state and binary action, and benefits from the improvement of the condition of the overall population. Based on an infinite horizon discounted individual cost, we show existence of a stationary equilibrium, and prove its uniqueness under a positive externality condition. We further analyze comparative statics of the stationary equilibrium by quantitatively determining the impact of the effort cost.
\end{abstract}

\section{Introduction}

Mean field game theory provides a powerful methodology for reducing complexity in the analysis and design of strategies in large population dynamic games [25, 30, 37. Following ideas in statistical physics, it takes a continuum approach to specify the aggregate impact of many individually insignificant players and solves a special stochastic optimal control problem from the point of view of a representative player. By this methodology, one may construct a set of decentralized strategies for the original large but finite population model and show its $\varepsilon$-Nash equilibrium property

Minyi Huang

School of Mathematics and Statistics, Carleton University, Ottawa, ON K1S 5B6, Canada e-mail: mhuang@math.carleton.ca. This author was supported by the Natural Sciences and Engineering Research Council (NSERC) of Canada

Yan Ma

School of Mathematics and Statistics, Zhengzhou University, 450001, Henan, China e-mail: mayan203ezzu.edu.cn. This author was supported by the National Science Foundation of China (No. 11601489)

* in IMA volume Modeling, Stochastic Control, Optimization, and Applications, eds. G. Yin and Q. Zhang, Springer, 2019, p. 283-313. Submitted Dec 2018; revised Feb 2019. This version: Oct 10, 2020. Minor changes in Example 2 
[25, 26, 30]. A related solution notion in Markov decision models is the oblivious equilibrium [55]. The readers are referred to [12, 16, 17, 18, 19] for an overview on mean field game theory and further references. For mean field type optimal control, see [12, 56], but the analysis in these models only involves a single decision maker.

Dynamic games within an MDP setting originated from the work of Shapley and are called stochastic games [21, 50]. Their mean field game extension has been studied in the literature; see e.g. [3, 13, 46, 55]. Continuous time mean field games with finite state space can be found in [22, 35]. Our previous work [27, 28] studied a class of mean field games in a multi-agent Markov decision process (MDP) framework. The players in [27] have continuum state spaces and binary action spaces, and have coupling through their costs. The state of each player is used to model its risk (or unfitness) level, which has random increase if no active control is taken. Naturally, the one-stage cost of a player is an increasing function of its own state apart from coupling with others. The motivation of this modeling framework comes from applications including network security investment games and flue vaccination games [34, 38, 40]; when the one-stage cost is an increasing function of the population average state, it reflects positive externalities. Markov decision processes with binary action spaces also arise in control of queues and machine replacement problems [4, 10]. Binary choice models have formed a subject of significant interest [8, 15, 48, 49, 54]. Our game model has connection with anonymous sequential games [33], which combine stochastic game modeling with a continuum of players. In anonymous sequential games one determines the equilibrium as a joint stateaction distribution of the population and leaves the individual strategies unspecified [33, Sec. 4], although there is an interpretation of randomized actions for players sharing a given state.

For both anonymous games and MDP based mean field games, stationary solutions with discount have been studied in the literature [3, 33]. These works give more focus on fixed point analysis to prove the existence of a stationary distribution. This approach does not address ergodic behavior of individuals or the population while assuming the population starts from the steady-state distribution at the initial time. Thus, there is a need to examine whether the individuals collectively have the ability to move into that distribution at all when they have a general initial distribution. Our ergodic analysis based approach will provide justification of the stationary solution regarding the population's ability to settle down around the limiting distribution.

The previous work [27, 28] studied the finite horizon mean field game by showing existence of a solution with threshold policies, and under an infinite horizon discounted cost further proved there is at most one stationary equilibrium for which existence was not established. A similar continuous time modeling is introduced in [57], which addresses Poisson state jumps and impulse control. It should be noted that except for linear-quadratic models [9, 26, 31, 39, 43], mean field games rarely have closed-form solutions and often rely on heavy numerical computations. Within this context, the consideration of structured solutions, such as threshold policies, is of particular interest from the point of view of efficient computation and simple implementation. Under such a policy, the individual states evolve as regenerative processes [6, 51]. 
By exploiting stochastic monotonicity, this paper adopts more general state transition assumptions than in [27, 28] and continues the analysis on the stationary equation system. The first contribution of the present paper is the proof of the existence of a stationary equilibrium. Our analysis depends on checking the continuous dependence of the limiting state distribution on the threshold parameter in the best response. The existence and uniqueness analysis in this paper has appeared in a preliminary form in the conference paper [29].

A key parameter in our game model is the effort cost. Intuitively, this parameter is a disincentive indicator of an individual for taking active efforts, and in turn will further impact the mean field forming the ambient environment of that agent. This suggests that we can study a family of mean field games parametrized by the effort costs and compare their solution behaviors. We address this in the setup of comparative statics, which have a long history in the economic literature [24, 42, 47] and operations research [53] and provide the primary means to analyze the effect of model parameter variations. For dynamic models, such as economic growth models, the analysis follows similar ideas and is sometimes called comparative dynamics [5, 11, 45, 47] by comparing two dynamic equilibria. In control and optimization, such studies are usually called sensitivity analysis [14, 20, 32]. For comparative statics in large static games and mean field games, see [1, 2]. Our analysis is accomplished by performing perturbation analysis around the equilibrium of the mean field game.

The paper is organized as follows. Section 2 introduces the mean field stochastic game. The best response is analyzed in Section 3. Section 4 proves existence and uniqueness of stationary equilibria. Comparative statics are analyzed in Section 5 , Section 6 concludes the paper.

\section{The Markov Decision Process Model}

\subsection{Dynamics and Costs}

The system consists of $N$ players denoted by $\mathscr{A}_{i}, 1 \leq i \leq N$. At time $t \in \mathbb{Z}_{+}=$ $\{0,1,2, \ldots\}$, the state of $\mathscr{A}_{i}$ is denoted by $x_{t}^{i}$, and its action by $a_{t}^{i}$. For simplicity, we consider a population of homogeneous (or symmetric) players. Each player has state space $\mathbf{S}=[0,1]$ and action space $\mathbf{A}=\left\{a_{0}, a_{1}\right\}$. A value of $\mathbf{S}$ may be interpreted as a risk or unfitness level. A player can either take inaction (as $a_{0}$ ) or make an active effort (as $\left.a_{1}\right)$. For an interval $I$, let $\mathscr{B}(I)$ denote the Borel $\sigma$-algebra of $I$.

The state of each player evolves as a controlled Markov process, which is affected only by its own action. For $t \geq 0$ and $x \in \mathbf{S}$, the state has a transition kernel specified by

$$
\begin{aligned}
& P\left(x_{t+1}^{i} \in B \mid x_{t}^{i}=x, a_{t}^{i}=a_{0}\right)=Q_{0}(B \mid x) \\
& P\left(x_{t+1}^{i}=0 \mid x_{t}^{i}=x, a_{t}^{i}=a_{1}\right)=1
\end{aligned}
$$


where $Q_{0}(\cdot \mid x)$ is a stochastic kernel defined for $B \in \mathscr{B}(\mathbf{S})$ and $Q_{0}([x, 1] \mid x)=1$. By the structure of $Q_{0}$, the state of the player deteriorates if no active control is taken. The vector process $\left(x_{t}^{1}, \ldots x_{t}^{N}\right)$ constitutes a controlled Markov process in higher dimension with its transition kernel defining a product measure on $(\mathscr{B}(\mathbf{S}))^{N}$ for given $\left(x_{t}^{1}, \cdots, x_{t}^{N}, a_{t}^{1}, \ldots, a_{t}^{N}\right)$.

Define the population average state $x_{t}^{(N)}=\frac{1}{N} \sum_{i=1}^{N} x_{t}^{i}$. The one stage cost of $\mathscr{A}_{i}$ is

$$
c\left(x_{t}^{i}, x_{t}^{(N)}, a_{t}^{i}\right)=R\left(x_{t}^{i}, x_{t}^{(N)}\right)+\gamma 1_{\left\{a_{t}^{i}=a_{1}\right\}},
$$

where $\gamma>0$ and $\gamma 1_{\left\{a_{t}^{i}=a_{1}\right\}}$ is the effort cost. The function $R \geq 0$ is defined on $\mathbf{S} \times \mathbf{S}$ and models the risk-related cost. Let $v^{i}$ denote the strategy of $\mathscr{A}_{i}$. We introduce the infinite horizon discounted cost

$$
J_{i}\left(x_{0}^{1}, \ldots, x_{0}^{N}, v^{1}, \ldots, v^{N}\right)=E \sum_{t=0}^{\infty} \beta^{t} c\left(x_{t}^{i}, x_{t}^{(N)}, a_{t}^{i}\right), \quad 1 \leq i \leq N .
$$

The standard methodology of mean field games may be applied by approximating $\left\{x_{t}^{(N)}, t \geq 0\right\}$ by a deterministic sequence $\left\{z_{t}, t \geq 0\right\}$ which depends on the initial condition of the system. One may solve the limiting optimal control problem of $\mathscr{A}_{i}$ and derive a dynamic programming equation for its value function denoted by $v_{i}\left(t, x,\left(z_{k}\right)_{k=0}^{\infty}\right)$, whose dependence on $t$ is due to the time-varying sequence $\left\{z_{t}, t \geq\right.$ $0\}$. Subsequently one derives another equation for the mean field $\left\{z_{t}, t \geq 0\right\}$ by averaging the individual states across the population. This approach, however, has the drawback of heavy computational load.

\subsection{Stationary Equilibrium}

We are interested in a steady-state form of the solution of the mean field game starting with $\left\{z_{t}, t \geq 0\right\}$. Such steady state equations provide information on the long time behavior of the solution and are of interest in their own right. They may also be used for approximation purposes to compute strategies efficiently. We introduce the system

$$
\begin{aligned}
& v(x)=\min \left[\beta \int_{0}^{1} v(y) Q_{0}(d y \mid x)+R(x, z), \quad \beta v(0)+R(x, z)+\gamma\right], \\
& z=\int_{0}^{1} x \mu(d x),
\end{aligned}
$$

where $\mu$ is a probability measure on $\mathbf{S}$. We say $\left(v, z, \mu, a^{i}(\cdot)\right)$ is a stationary equilibrium to (4)-(5) if i) the feedback policy $a^{i}(\cdot)$, as a mapping from $\mathbf{S}$ to $\left\{a_{0}, a_{1}\right\}$, is the best response with respect to $z$ in (4), ii) given an initial distribution of $x_{0}^{i},\left\{x_{t}^{i}, t \geq 0\right\}$ under the policy $a^{i}$ has its distribution converging (under a total variation norm or only weakly) to the stationary distribution (or called limiting distribution) $\mu$. 
We may interpret $v$ as the value function of an MDP with cost $\bar{J}_{i}\left(x_{0}^{i}, z, v^{i}\right)=$ $E \sum_{t=0}^{\infty} \beta^{t} c\left(x_{t}^{i}, z, a_{t}^{i}\right)$. An alternative way to interpret (4)-(5) is that the initial state of $\mathscr{A}_{i}$ has been sampled according to the "right" distribution $\mu$, and that $z$ is obtained by averaging an infinite number of such initial values by the law of large numbers [52]. A similar solution notion is adopted in [2, 3] but ergodicity is not part of their solution specification.

Let the probability measure $\mu_{k}$ be the distribution of $\mathbb{R}$-valued random variable $Z_{k}, k=1,2$. We say $\mu_{2}$ stochastically dominates $\mu_{1}$, and denote $\mu_{1} \leq_{s t} \mu_{2}$, if $\mu_{2}((y, \infty)) \geq \mu_{1}\left((y, \infty)\right.$ ) (or equivalently, $P\left(Z_{2}>y\right) \geq P\left(Z_{1}>y\right)$ ) for all $y$. It is well known [44] that $\mu_{1} \leq_{s t} \mu_{2}$ if and only if

$$
\int \psi(y) \mu_{1}(d y) \leq \int \psi(y) \mu_{2}(d y)
$$

for all increasing function $\psi$ (not necessarily strictly increasing) for which the two integrals are finite. A stochastic kernel $\mathscr{Q}(B \mid x), 0 \leq x \leq 1, B \in \mathscr{B}(\mathbf{S})$, is said to be strictly stochastically increasing if $\varphi(x):=\int_{\mathbf{S}} \psi(y) \mathscr{Q}(d y \mid x)$ is strictly increasing in $x \in \mathbf{S}$ for any strictly increasing function $\psi:[0,1] \rightarrow \mathbb{R}$ for which the integral is necessarily finite. $\mathscr{Q}(\cdot \mid x)$ is said to be weakly continuous if $\varphi$ is continuous whenever $\psi$ is continuous.

Let $\left\{Y_{t}, t \geq 0\right\}$ be a Markov process with state space $[0,1]$, transition kernel $Q_{0}(\cdot \mid x)$ and initial state $Y_{0}=0$. So each of its trajectories is monotonically increasing. Define $\tau_{Q_{0}}^{\theta}=\inf \left\{t \mid Y_{t} \geq \theta\right\}$ for $\theta \in(0,1)$. It is clear that $\tau_{Q_{0}}^{\theta_{1}} \leq \tau_{Q_{0}}^{\theta_{2}}$ for $0<\theta_{1}<\theta_{2}<1$.

The following assumptions are introduced.

(A1) $\quad\left\{x_{0}^{i}, i \geq 1\right\}$ are i.i.d. random variables taking values in $\mathbf{S}$.

(A2) $R(x, z)$ is a continuous function on $\mathbf{S} \times \mathbf{S}$. For each fixed $z, R(\cdot, z)$ is strictly increasing.

(A3) i) $Q_{0}(\cdot \mid x)$ satisfies $Q_{0}([x, 1] \mid x)=1$ for any $x$, and is strictly stochastically increasing; ii) $Q_{0}(d y \mid x)$ is weakly continuous and has a positive probability density $q(y \mid x)$ for each fixed $x<1$; iii) for any small $0<\delta<1$, $\inf _{x} Q_{0}([1-\delta, 1] \mid x)>0$.

(A4) $R(x, \cdot)$ is increasing for each fixed $x$.

(A5) $\lim _{\theta \uparrow 1} E \tau_{Q_{0}}^{\theta}=\infty$.

(A3)-iii) will be used to ensure the uniform ergodicity of the controlled Markov process. In fact, under (A3) we can show $E \tau_{Q_{0}}^{\theta}<\infty$. The following condition is a special case of (A3).

$\left(\mathrm{A} 3^{\prime}\right)$ There exists a random variable such that $Q_{0}(\cdot \mid x)$ is equal to the law of $x+(x-1) \xi$ for some random variable $\xi$ with probability density $f_{\xi}(x)>0$, a.e. $x \in \mathbf{S}$.

When (A3') holds, we can verify (A5) by analyzing the stopping time $\tau_{\xi}=$ $\inf \left\{t \mid \prod_{s=1}^{t} \xi_{s} \leq 1-\theta\right\}$, where $\left\{\xi_{s}, s \geq 1\right\}$ is a sequence of i.i.d. random variables with probability density $f_{\xi}$. For existence analysis of the mean field game, (A5) will be used to ensure continuity of the mean field when the threshold $\theta$ approaches 1 . 
Proposition 1 The two conditions are equivalent:

i) $\mu_{1} \leq_{s t} \mu_{2}$, and $\mu_{1} \neq \mu_{2}$;

ii) $\int_{\mathbb{R}} \phi(y) \mu_{1}(d y)<\int_{\mathbb{R}} \phi(y) \mu_{2}(d y)$ for all strictly increasing function $\phi$ for which both integrals are finite.

Proof. Assume i) holds. By [44, Theorem 1.2.16], we have

$$
\phi\left(Z_{1}\right) \leq_{s t} \phi\left(Z_{2}\right)
$$

and so $E \phi\left(Z_{1}\right) \leq E \phi\left(Z_{2}\right)$. Since $\mu_{1} \neq \mu_{2}$, there exists $y_{0}$ such that $P\left(Z_{1}>y_{0}\right) \neq$ $P\left(Z_{2}>y_{0}\right)$. Take $r$ such that $\phi\left(y_{0}\right)=r$. Then

$$
P\left(\phi\left(Z_{1}\right)>r\right) \neq P\left(\phi\left(Z_{2}\right)>r\right) .
$$

If $E \phi\left(Z_{1}\right)=E \phi\left(Z_{2}\right)$ were true, by (7) and [44, Theorem 1.2.9], $\phi\left(Z_{1}\right)$ and $\phi\left(Z_{2}\right)$ would have the same distribution, which contradicts (8). We conclude $E \phi\left(Z_{1}\right)<$ $E \phi\left(Z_{2}\right)$, which is equivalent to ii).

Next we show ii) implies i). Let $\psi$ be any increasing function satisfying (6) with two finite integrals. When ii) holds, we take $\phi_{\varepsilon}=\psi+\frac{\varepsilon y}{1+|y|}, \varepsilon>0$. Then $\int \phi_{\varepsilon} \mu_{1}(d y)<\int \phi_{\varepsilon} \mu_{2}(d y)$ holds for all $\varepsilon>0$. Letting $\varepsilon \rightarrow 0$, then (6) follows and $\mu_{1} \leq_{s t} \mu_{2}$. It is clear $\mu_{1} \neq \mu_{2}$.

\section{Best Response}

For this section we assume (A1)-(A3). We take any fixed $z \in[0,1]$ and consider (4) as a separate equation, which is rewritten below:

$$
v(x)=\min \left\{\beta \int_{0}^{1} v(y) Q_{0}(d y \mid x)+R(x, z), \quad \beta v(0)+R(x, z)+\gamma\right\} .
$$

Here $z$ is not required to satisfy (5). In relation to the mean field game, the resulting optimal policy will be called the best response with respect to $z$. Denote $G(x)=$ $\int_{0}^{1} v(y) Q_{0}(d y \mid x)$.

Lemma 1. i) Equation (9] has a unique solution $v \in C([0,1], \mathbb{R})$.

ii) $v$ is strictly increasing.

iii) The optimal policy is determined as follows:

a) If $\beta G(1)<\beta v(0)+\gamma, a^{i}(x) \equiv a_{0}$.

b) If $\beta G(1)=\beta v(0)+\gamma, a^{i}(1)=a_{1}$ and $a^{i}(x)=a_{0}$ for $x<1$.

c) If $\beta G(0) \geq \beta v(0)+\gamma, a^{i}(x) \equiv a_{1}$.

d) If $\beta G(0)<\beta v(0)+\gamma<\rho G(1)$, there exists a unique $x^{*} \in(0,1)$ and $a^{i}$ is a threshold policy with parameter $x^{*}$, i.e., $a^{i}(x)=a_{1}$ if $x \geq x^{*}$ and $a^{i}(x)=a_{0}$ if $x<x^{*}$.

Proof. Define the dynamic programming operator 


$$
(\mathscr{L} g)(x)=\min \left\{\beta \int_{0}^{1} g(y) Q_{0}(d y \mid x)+R(x, z), \quad \beta g(0)+R(x, z)+\gamma\right\},
$$

which is from $C([0,1], \mathbb{R})$ to itself. The proving method in [27], [28, Lemma 6], which assumed $\left(\mathrm{A}^{\prime}\right)$, can be extended to the present equation (9) in a straightforward manner.

In particular, for the proof of ii) and iii), we obtain progressively stronger properties of $v$ and $G$. First, denoting $g_{0}=0$ and $g_{k+1}=\mathscr{L} g_{k}$ for $k \geq 0$, we use a successive approximation procedure to show that $v$ is increasing, which implies that $G$ is continuous and increasing by weak continuity and monotonicity of $Q_{0}$. Since $R$ is strictly increasing in $x$, by the right hand side of (9), we show that $v$ is strictly increasing, which implies the same property for $G$ by strict monotonicity of $Q_{0}$.

For the optimal policy specified in part iii) of Lemma 1, we can formally denote the threshold parameters for the corresponding cases: a) $\theta=1^{+}$, b) $\theta=1$, c) $\theta=$ 0 , and d) $\theta=x^{*}$. Such a policy will be called a $\theta$-threshold policy. We give the condition for $\theta=0$ in the best response.

Lemma 2. For $\gamma>0$ and $v$ solving (9),

$$
\beta G(0) \geq \beta v(0)+\gamma
$$

holds if and only if

$$
\gamma \leq \beta \int_{0}^{1} R(y, z) Q_{0}(d y \mid 0)-\beta R(0, z) .
$$

Proof. We show necessity first. Suppose (11) holds. Note that $G(x)$ is strictly increasing on $[0,1]$. Equation (9) reduces to

$$
\begin{aligned}
& v(x)=\beta v(0)+R(x, z)+\gamma \\
& \beta G(x) \geq \beta v(0)+\gamma, \quad \forall x .
\end{aligned}
$$

From (13), we uniquely solve

$$
v(0)=\frac{1}{1-\beta}[R(0, z)+\gamma], \quad v(x)=\frac{\beta}{1-\beta}[R(0, z)+\gamma]+R(x, z)+\gamma,
$$

which combined with (14) implies (12).

We continue to show sufficiency. If $\gamma>0$ satisfies (12), we use (15) to construct $v$ and verify (13) and (14). So $v$ is the unique solution of (9) satisfying (11).

The next lemma gives the condition for $\theta=1^{+}$in the best response.

Lemma 3. For $\gamma>0$ and $v$ solving (9), we have

$$
\beta G(1)<\beta v(0)+\gamma
$$

if and only if 


$$
\gamma>\beta\left[V_{\beta}(1)-V_{\beta}(0)\right]
$$

where $V_{\beta}(x) \in C([0,1], \mathbb{R})$ is the unique solution of

$$
V_{\beta}(x)=\beta \int_{0}^{1} V_{\beta}(y) Q_{0}(d y \mid x)+R(x, z) .
$$

Proof. By Banach's fixed point theorem, we can show that 18 has a unique solution. Next, by a successive approximation $\left\{V_{\beta}^{(k)}, k \geq 0\right\}$ with $V_{\beta}^{(0)}=0$ in the fixed point equation, we can further show that $V_{\beta}$ is strictly increasing. Moreover, $\int_{0}^{1} V_{\beta}(y) Q_{0}(d y \mid x)$ is increasing in $x$ by monotonicity of $Q_{0}$.

We show necessity. Since $G$ is strictly increasing, (16) implies that the right hand side of (9) now reduces to the first term within the parentheses and that $v=V_{\beta}$. So (17) follows.

To show sufficiency, suppose 17) holds. We have

$$
\beta \int_{0}^{1} V_{\beta}(y) Q_{0}(d y \mid x) \leq \beta V_{\beta}(1)<\beta V_{\beta}(0)+\gamma, \quad \forall x .
$$

Therefore, $v:=V_{\beta}$ gives the unique solution of (9) and $\beta G(1)<\beta v(0)+\gamma$.

Example 1. Let $R(x, z)=x(c+z)$, where $c>0$. Take $Q_{0}(\cdot \mid x)$ as uniform distribution on $[x, 1]$. Then $(18)$ reduces to

$$
V_{\beta}(x)=\frac{\beta}{1-x} \int_{x}^{1} V_{\beta}(y) d y+R(x, z) .
$$

Define $\phi(x)=\int_{x}^{1} V_{\beta}(y) d y, x \in[0,1]$. Then $\phi^{\prime}(x)=-\frac{\beta}{1-x} \phi(x)-R(x, z)$ holds and we solve

$$
\phi(x)=(1-x)^{\beta} \int_{x}^{1} \frac{R(s, z)}{(1-s)^{\beta}} d s,
$$

where the right hand side converges to 0 as $x \rightarrow 1^{-}$. We further obtain

$$
V_{\beta}(x)=\beta(1-x)^{\beta-1} \int_{x}^{1} \frac{R(s, z)}{(1-s)^{\beta}} d s+R(x, z)
$$

for $x \in[0,1)$, and the right hand side has the limit $\frac{R(1, z)}{1-\beta}$ as $x \rightarrow 1^{-}$. This gives a well defined $V_{\beta} \in C([0,1], \mathbb{R})$. Therefore, $V_{\beta}(0)=\frac{\beta(c+z)}{(1-\beta)(2-\beta)}$. Then (17) reduces to $\gamma>\frac{2 \beta(c+z)}{2-\beta}$. 


\section{Existence of Stationary Equilibria}

Assume (A1)-(A5) for this section. Define the class $\mathscr{P}_{0}$ of probability measures on $\mathbf{S}$ as follows: $v \in \mathscr{P}_{0}$ if there exist a constant $c_{v} \geq 0$ and a Borel measurable function $g(x) \geq 0$ defined on $[0,1]$ such that

$$
v(B)=\int_{B} g(x) d x+c_{V} 1_{B}(0),
$$

where $B \in \mathscr{B}(\mathbf{S})$ and $1_{B}$ is the indicator function of $B$. When restricted to $(0,1], v$ is absolutely continuous with respect to the Lebesgue measure $\mu^{\mathrm{Leb}}$.

Let $X$ be a random variable with distribution $v \in \mathscr{P}_{0}$. Set $x_{t}^{i}=X$. Define $Y_{0}=x_{t+1}^{i}$ by applying $a_{t}^{i} \equiv a_{0}$. Further define $Y_{1}=x_{t+1}^{i}$ by applying the $r$-threshold policy $a_{t}^{i}$ with $r \in(0,1)$.

Lemma 4. The distribution $v_{i}$ of $Y_{i}$ is in $\mathscr{P}_{0}$ for $i=0,1$.

Proof. Let $q(y \mid x)$ denote the density function of $Q_{0}(\cdot \mid x)$ for $x \in[0,1)$, where $q(y \mid x)=0$ for $y<x$. Denote

$$
g_{0}(y)=\int_{0 \leq x<y} q(y \mid x) v(d x), \quad y \in(0,1)
$$

and

$$
g_{1}(y)=\int_{0 \leq x<y \wedge r} q(y \mid x) v(d x), \quad y \in(0,1) .
$$

Then it can be checked that

$$
P\left(Y_{0} \in B\right)=\int_{B} g_{0}(y) d y, \quad P\left(Y_{1} \in B\right)=\int_{B} g_{1}(y) d y+P(X \geq r) 1_{B}(0) .
$$

This completes the lemma.

In order to show that (4)-(5) has a solution, we define a mapping $\Gamma: \mathbf{S} \rightarrow \mathbf{S}$ by the following rule. For $z \in[0,1]$, we solve (4) to obtain a well defined threshold $\theta(z) \in[0,1] \cup\left\{1^{+}\right\}$, which in turn determines a limiting distribution $\mu_{\theta(z)}$ of the closed-loop state process $x_{t}^{i}$ by Lemma A.1 Define

$$
\Gamma(z)=\int_{0}^{1} x \mu_{\theta(z)}(d x) .
$$

If $\Gamma$ has a fixed point, we obtain a solution to (4)-(5).

We analyze the case where the best response gives a strictly positive threshold. Assume

$$
\gamma>\beta \max _{z \in[0,1]} \int_{0}^{1}[R(y, z)-R(0, z)] Q_{0}(d y \mid 0)
$$


Note that under a zero threshold policy, the behavior of the state process is sensitive to a positive perturbation of the threshold. The above condition ensures that the zero threshold will not occur, and this will ensure continuity of $\Gamma$ to facilitate the fixed point analysis.

Lemma 5. Assume (19). Then $\Gamma(z)$ is continuous on $[0,1]$.

Proof. Let $z_{0} \in[0,1]$ be fixed, giving a corresponding threshold parameter $\theta_{0}$ when (9) is solved using $z_{0}$. We check continuity at $z_{0}$ and consider 3 cases.

Case i) $\theta_{0} \in(0,1)$. Let $\pi_{0}$ be the stationary distribution with the $\theta_{0}$-threshold policy. Consider any fixed $\varepsilon>0$. There exists $\varepsilon_{1}$ such that for all $\theta \in\left(\theta_{0}-\varepsilon_{1}, \theta_{0}+\right.$ $\left.\varepsilon_{1}\right) \subset(0,1),\left|\int_{0}^{1} x \pi(d x)-\int_{0}^{1} x \pi_{0}(d x)\right|<\varepsilon$, where $\pi$ is the stationary distribution associated with $\theta$. This follows since $\lim _{\theta \rightarrow \theta_{0}}\left\|\pi-\pi_{0}\right\|_{\mathrm{TV}}=0$ by Lemma A.3. Now by the continuous dependence of the solution of the dynamic programming equation on $z$, we can select a sufficiently small $\delta>0$ such that for all $\left|z-z_{0}\right|<\delta$, $z$ generates a threshold parameter $\theta \in\left(\theta_{0}-\varepsilon_{1}, \theta_{0}+\varepsilon_{1}\right)$, which implies $\left|\Gamma(z)-\Gamma\left(z_{0}\right)\right| \leq \varepsilon$.

Case ii) $z_{0}$ gives $\theta_{0}=1$. Then $\Gamma\left(z_{0}\right)=1$. Fix any $\varepsilon>0$. Then we can show there exists $\varepsilon_{1}$ such that for all $\theta \in\left(1-\varepsilon_{1}, 1\right)$, the associated stationary distribution $\pi_{\theta}$ gives $\left|\Gamma\left(z_{0}\right)-\int_{0}^{1} x \pi_{\theta}(d x)\right|<\varepsilon$, where we use (A5) and the right hand side of (C.1) to estimate a lower bound for $\int_{0}^{1} x \pi_{\theta}(d x)$. Now, there exists $\delta>0$ such that any $z$ satisfying $\left|z-z_{0}\right|<\delta$ gives a threshold $\theta$ either in $\left(1-\varepsilon_{1}, 1\right)$ or equal to 1 or $1^{+}$; for each case, we have $\left|\Gamma\left(z_{0}\right)-\int_{0}^{1} x \pi_{\theta}(d x)\right|<\varepsilon$.

Case iii) $z_{0}$ gives $\theta_{0}=1^{+}$. Then there exists $\delta>0$ such that any $z$ satisfying $\left|z-z_{0}\right|<\delta$ gives a threshold parameter $\theta=1^{+}$. Then $\Gamma(z)=\Gamma\left(z_{0}\right)=1$.

Theorem 1. Assume (19). There exists a stationary equilibrium to (4)-(5).

Proof. Since $\Gamma$ is a continuous function from $[0,1]$ to $[0,1]$ by Lemma 5 , the theorem follows from Brouwer's fixed point theorem.

Let $x_{t}^{i, \theta}$ and $\pi_{\theta}$ denote the state process and its stationary distribution, respectively, under a $\theta$-threshold policy. Denote $z(\theta)=\int_{0}^{1} x \pi_{\theta}(d x)$. We have the first comparison theorem on monotonicity.

Lemma 6. $z\left(\theta_{1}\right) \leq z\left(\theta_{2}\right)$ for $0<\theta_{1}<\theta_{2}<1$.

Proof. By the ergodicity of $\left\{x_{t}^{i, \theta_{l}}, t \geq 0\right\}$ in LemmaA.2, we have the representation $z\left(\theta_{l}\right)=\lim _{k \rightarrow \infty} \frac{1}{k} \sum_{t=0}^{k-1} x_{t}^{i, \theta_{l}}$ w.p.1. LemmaC.2 implies $z\left(\theta_{1}\right) \leq z\left(\theta_{2}\right)$.

To establish uniqueness, we consider $R(x, z)=R_{1}(x) R_{2}(z)$, where $R_{1} \geq 0$ and $R_{2} \geq 0$, and which satisfies (A1)-(A5). We further make the following assumption.

$$
R_{2}>0 \text { is strictly increasing on } \mathbf{S} \text {. }
$$

This assumption indicates positive externalities since an individual benefits from the decrease of the population average state. This condition has a crucial role in the uniqueness analysis.

Given the product form of $R$, now (9) takes the form: 


$$
V(x)=\min \left[\beta \int_{0}^{1} V(y) Q_{0}(d y \mid x)+R_{1}(x) R_{2}(z), \quad \beta V(0)+R_{1}(x) R_{2}(z)+\gamma\right] .
$$

Consider $0 \leq z_{2}<z_{1} \leq 1$ and

$$
V_{l}(x)=\min \left[\beta \int_{0}^{1} V_{l}(y) Q_{0}(d y \mid x)+R_{1}(x) R_{2}\left(z_{l}\right), \quad \beta V_{l}(0)+R_{1}(x) R_{2}\left(z_{l}\right)+\gamma\right] .
$$

Denote the optimal policy as a threshold policy with parameter $\theta_{l}$ in $[0,1]$ or equal to $1^{+}$, where we follow the interpretation in Section 3 if $\theta_{l}=1^{+}$. We state the second comparison theorem about the threshold parameters under different mean field parameters $z_{l}$.

Theorem 2. $\theta_{1}$ and $\theta_{2}$ in (20) are specified according to the following scenarios:

i) If $\theta_{1}=0$, then we have either $\theta_{2} \in[0,1]$ or $\theta_{2}=1^{+}$.

ii) If $\theta_{1} \in(0,1)$, we have either a) $\theta_{2} \in\left(\theta_{1}, 1\right)$, or b) $\theta_{2}=1$, or c) $\theta_{2}=1^{+}$.

iii) If $\theta_{1}=1, \theta_{2}=1^{+}$.

iv) If $\theta_{1}=1^{+}, \theta_{2}=1^{+}$.

Proof. Since $R_{2}\left(z_{1}\right)>R_{2}\left(z_{2}\right)>0$, we divide both sides of (20) by $R_{2}\left(z_{l}\right)$ and define $\gamma_{l}=\frac{\gamma}{R_{2}\left(z_{l}\right)}$. Then $0<\gamma_{1}<\gamma_{2}$. The dynamic programming equation reduces to (D.2). Subsequently, the optimal policy is determined according to Lemma D.4.

Corollary 1. Assume (A6) in addition to the assumptions in Theorem 1 Then the system (4)-(5) has a unique stationary equilibrium.

Proof. The proof is similar to [27, 28], which assumed (A3').

\section{Comparative Statics}

This section assumes (A1)-(A6). Consider the two solution systems

$$
\left\{\begin{array}{l}
\bar{v}(x)=\min \left[\beta \int_{0}^{1} \bar{v}(y) Q_{0}(d y \mid x)+R_{1}(x) R_{2}(\bar{z}), \quad \beta \bar{v}(0)+R_{1}(x) R_{2}(\bar{z})+\bar{\gamma}\right], \\
\bar{z}=\int_{0}^{1} x \bar{\mu}(d x),
\end{array}\right.
$$

and

$$
\left\{\begin{array}{l}
v(x)=\min \left[\beta \int_{0}^{1} v(y) Q_{0}(d y \mid x)+R_{1}(x) R_{2}(z), \quad \beta v(0)+R_{1}(x) R_{2}(z)+\gamma\right], \\
z=\int_{0}^{1} x \mu(d x) .
\end{array}\right.
$$


Suppose $\bar{\gamma}$ satisfies (19). By Corollary 11 (21) has a unique solution denoted by $(\bar{v}, \bar{z}, \bar{\mu}, \bar{\theta})$, where $\bar{\theta}$ is the threshold parameter. We further assume $\bar{\theta} \in(0,1)$. Suppose $\gamma>\bar{\gamma}$. Then we can uniquely solve $(v, z, \mu, \theta)$. The next theorem presents a result on monotone comparative statics [53].

Theorem 3. If $\gamma>\bar{\gamma}$, we have

$$
\theta>\bar{\theta}, \quad z>\bar{z}, \quad v>\bar{v} .
$$

Proof. We prove by contradiction. Assume $\theta \leq \bar{\theta}$. Then by Lemma $6, z \leq \bar{z}$, and therefore, $\frac{\gamma}{R_{2}(z)}>\frac{\bar{\gamma}}{R_{2}(\bar{z})}$. By the method of proving Theorem 2, we would establish $\theta>\bar{\theta}$, which contradicts the assumption $\theta \leq \bar{\theta}$. We conclude $\theta>\bar{\theta}$. By Lemma 6 and Remark B.1, we have $z>\bar{z}$. For (21), we use value iteration to approximate $\bar{v}$ by an increasing sequence of functions $\bar{v}_{k}$ with $\bar{v}_{0}=0$. Similarly, $v$ is approximated by $v_{k}$ with $v_{0}=0$. By induction, we have $v_{k} \geq \bar{v}_{k}$ for all $k$. This proves $v \geq \bar{v}$.

Next, we have $\beta v(0)+R_{1}(x) R_{2}(z)+\gamma>\beta \bar{v}(0)+R_{1}(x) R_{2}(\bar{z})+\bar{\gamma}$ on $[0,1]$, and $\beta \int_{0}^{1} v(y) Q_{0}(d y \mid x)+R_{1}(x) R_{2}(z)>\beta \int_{0}^{1} \bar{v}(y) Q_{0}(d y \mid x)+R_{1}(x) R_{2}(\bar{z})$ on $(0,1]$. By the method in [27, Lemma 2], we have $v>\bar{v}$ on $(0,1]$. Then $\int_{0}^{1} v(y) Q_{0}(d y \mid 0)>$ $\int_{0}^{1} \bar{v}(y) Q_{0}(d y \mid 0)$. This further implies $v(0)>\bar{v}(0)$.

Remark 1. It is possible to have $\theta=1^{+}$in Theorem 3

By a continuity argument, we can further show $\lim _{\gamma \rightarrow \bar{\gamma}}(|\theta-\bar{\theta}|+|z-\bar{z}|+$ $\left.\sup _{x}|v(x)-\bar{v}(x)|\right)=0$. In the analysis below, we take $\gamma=\bar{\gamma}+\varepsilon$ for some small $\varepsilon>0$. For this section, we further introduce the following assumption.

For $\gamma>\bar{\gamma},(v, z, \theta)$ has the representation

$$
\begin{aligned}
& v(x)=\bar{v}(x)+\varepsilon w(x)+o(\varepsilon), \quad 0 \leq x \leq 1, \\
& z=\bar{z}+\varepsilon z_{\gamma}+o(\varepsilon), \\
& \theta=\bar{\theta}+\varepsilon \theta_{\gamma}+o(\varepsilon),
\end{aligned}
$$

where $v, z, \theta$ are solved depending on the parameter $\gamma$ and $w$ is a function defined on $[0,1]$. The derivatives $z_{\gamma}$ and $\theta_{\gamma}$ at $\bar{\gamma}$ exist, and $R_{2}(z)$ is differentiable on $[0,1]$. For $0 \leq x<1$, the probability density function $q(y \mid x), y \in[x, 1]$, for $Q_{0}(d y \mid x)$ is continuous on $\{(x, y) \mid 0 \leq x \leq y<1\}$. Moreover, $\frac{\partial q(y \mid x)}{\partial x}$ exists and is continuous in $(x, y)$.

We aim to provide a characterization of $w, z_{\gamma}, \theta_{\gamma}$.

Theorem 4. The function $w$ satisfies

$$
w(x)= \begin{cases}\beta \int_{0}^{1} w(y) Q_{0}(d y \mid x)+R_{1}(x) R_{2}^{\prime}(\bar{z}) z_{\gamma}, & 0 \leq x \leq \bar{\theta}, \\ \beta w(0)+R_{1}(x) R_{2}^{\prime}(\bar{z}) z_{\gamma}+1, & \bar{\theta}<x \leq 1 .\end{cases}
$$

Proof. We have 


$$
\bar{v}(x)=\beta \int_{0}^{1} \bar{v}(y) Q_{0}(d y \mid x)+R_{1}(x) R_{2}(\bar{z}), \quad x \in[0, \bar{\theta}]
$$

and

$$
v(x)=\beta \int_{0}^{1} v(y) Q_{0}(d y \mid x)+R_{1}(x) R_{2}(z), \quad x \in[0, \theta] .
$$

Note that $\theta>\bar{\theta}$. For any fixed $x \in[0, \bar{\theta}]$, we have

$$
v(x)-\bar{v}(x)=\beta \int_{0}^{1}(v(y)-\bar{v}(y)) Q_{0}(d y \mid x)+R_{1}(x)\left(R_{2}(z)-R_{2}(\bar{z})\right) .
$$

Then the equation of $w(x)$ for $x \in[0, \bar{\theta}]$ is derived. We similarly treat the case $x \in$ $(\bar{\theta}, 1]$.

Remark 2. In general $w$ has discontinuity at $x=\bar{\theta}$, so that $\beta \int_{0}^{1} w(y) Q_{0}(d y \mid \bar{\theta}) \neq$ $\beta w(0)+1$. We give some interpretation. Let the value function be written as $v(x, \gamma)$ to explicitly indicate $\gamma$. Let the rectangle $[0,1] \times\left[\gamma_{a}, \gamma_{b}\right]$ be a region of interest in which $(x, \gamma)$ varies so that the value function defines a continuous surface. Then $(\theta, \gamma)$ starts at $(\bar{\theta}, \bar{\gamma})$ and traces out the curve of an increasing function along which the expression of the value function has a switch, and the value function surface may be visualized as two pieces glued together along the curve in a non-smooth way. The value of $w$ amounts to finding on the surface the directional derivative in the direction of $\gamma$; and therefore, discontinuity may occur at $x=\bar{\theta}$.

To better understand the solution of (26), we consider the general equation

$$
W(x)= \begin{cases}\beta \int_{0}^{1} W(y) Q_{0}(d y \mid x)+R_{1}(x) R_{2}^{\prime}\left(z_{0}\right) c_{0}, & 0 \leq x \leq \theta_{0}, \\ \beta W(0)+R_{1}(x) R_{2}^{\prime}\left(z_{0}\right) c_{0}+1, & \theta_{0}<x \leq 1,\end{cases}
$$

where $c_{0}, z_{0} \in[0,1]$ and $\theta_{0} \in(0,1)$ are arbitrarily chosen and fixed. Let $B([0,1], \mathbb{R})$ be the Banach space of bounded Borel measurable functions with norm $\|g\|=$ $\sup _{x}|g(x)|$. By a contraction mapping, we can show 27) has a unique solution $W \in B([0,1], \mathbb{R})$.

We continue to characterize the sensitivity $\theta_{\gamma}$ of the threshold. Recall the partial derivative $\frac{\partial q(y \mid x)}{\partial x}$.

Lemma 7. We have

$$
\beta\left[\int_{\bar{\theta}}^{1} \bar{v}(y) \frac{\partial q(y \mid \bar{\theta})}{\partial x} d y-\bar{v}(\bar{\theta}) q(\bar{\theta} \mid \bar{\theta})\right] \theta_{\gamma}=1+\beta w(0)-\beta \int_{\bar{\theta}}^{1} w(y) Q_{0}(d y \mid \bar{\theta}) .
$$

Proof. Write $\gamma=\bar{\gamma}+\varepsilon$. By the property of the threshold, we have

$$
\beta \int_{\bar{\theta}}^{1} \bar{v}(y) Q_{0}(d y \mid \bar{\theta})=\beta \bar{v}(0)+\bar{\gamma}, \quad \beta \int_{\theta}^{1} v(y) Q_{0}(d y \mid \theta)=\beta v(0)+\bar{\gamma}+\varepsilon .
$$


Note that $\theta>\bar{\theta}$. We check

$$
\begin{aligned}
\Delta:= & \int_{\theta}^{1} v(y) Q_{0}(d y \mid \theta)-\int_{\bar{\theta}}^{1} \bar{v}(y) Q_{0}(d y \mid \bar{\theta}) \\
= & \int_{\theta}^{1} v(y) Q_{0}(d y \mid \theta)-\int_{\theta}^{1} \bar{v}(y) Q_{0}(d y \mid \bar{\theta})-\int_{\bar{\theta}}^{\theta} \bar{v}(y) Q_{0}(d y \mid \bar{\theta}) \\
= & \int_{\theta}^{1} v(y) Q_{0}(d y \mid \theta)-\int_{\theta}^{1} \bar{v}(y) Q_{0}(d y \mid \theta) \\
& +\int_{\theta}^{1} \bar{v}(y) Q_{0}(d y \mid \theta)-\int_{\theta}^{1} \bar{v}(y) Q_{0}(d y \mid \bar{\theta})-\int_{\bar{\theta}}^{\theta} \bar{v}(y) Q_{0}(d y \mid \bar{\theta}) \\
= & \varepsilon \int_{\theta}^{1} w(y) q(y \mid \theta) d y+(\theta-\bar{\theta}) \int_{\theta}^{1} \bar{v}(y)[\partial q(y \mid \theta) / \partial x] d y-(\theta-\bar{\theta}) \bar{v}(\bar{\theta}) q(\bar{\theta} \mid \bar{\theta}) \\
& +o(\varepsilon+|\theta-\bar{\theta}|) \\
= & \varepsilon \int_{\bar{\theta}}^{1} w(y) q(y \mid \bar{\theta}) d y+(\theta-\bar{\theta}) \int_{\bar{\theta}}^{1} \bar{v}(y)[\partial q(y \mid \bar{\theta}) / \partial x] d y-(\theta-\bar{\theta}) \bar{v}(\bar{\theta}) q(\bar{\theta} \mid \bar{\theta}) \\
& +o(\varepsilon+|\theta-\bar{\theta}|) .
\end{aligned}
$$

Note that

$$
\beta \Delta=\beta[v(0)-\bar{v}(0)]+\varepsilon .
$$

We derive

$$
\beta \int_{\bar{\theta}}^{1} w(y) Q_{0}(d y \mid \bar{\theta})+\beta \theta_{\gamma} \int_{\bar{\theta}}^{1} \bar{v}(y) \frac{\partial q(y \mid \bar{\theta})}{\partial x} d y-\beta \bar{v}(\bar{\theta}) q(\bar{\theta} \mid \bar{\theta}) \theta_{\gamma}=\beta w(0)+1 .
$$

This completes the proof.

Lemma 8. Given the threshold $\overline{\boldsymbol{\theta}} \in(0,1)$, the stationary distribution $\bar{\mu}$ has a probability density function (p.d.f.) $p(x)$ on $(0,1]$, and $\bar{\mu}(\{0\})=\pi_{0}$, where $\left(p, \pi_{0}\right)$ is determined by

$$
\begin{aligned}
& \pi_{0}=\int_{\bar{\theta}}^{1} p(x) d x, \\
& p(x)= \begin{cases}\int_{0}^{x} q(x \mid y) p(y) d y+\pi_{0} q(x \mid 0), & 0 \leq x<\bar{\theta}, \\
\int_{0}^{\bar{\theta}} q(x \mid y) p(y) d y+\pi_{0} q(x \mid 0), & \bar{\theta} \leq x \leq 1 .\end{cases}
\end{aligned}
$$

Proof. Let $\delta_{0}$ be the dirac measure at $x=0$. For any Borel subset $B \subset[0,1]$, we have $\bar{\mu}(B)=\int_{0}^{1}\left[Q_{0}(B \mid y) 1_{(y<\bar{\theta})}+\delta_{0}(B) 1_{(y \geq \bar{\theta})}\right] \bar{\mu}(d y)$. Then it can be checked that $\left(p, \pi_{0}\right)$ satisfying the above equations determines the stationary distribution. Now we show there exists a unique solution. Let $\pi_{0}>0$ be a constant to be determined. Consider the Volterra integral equation

$$
p(x)=\int_{0}^{x} q(x \mid y) p(y) d y+\pi_{0} q(x \mid 0), \quad 0 \leq x \leq \bar{\theta},
$$


and we obtain a unique solution $p$ in $C([0, \bar{\theta}], \mathbb{R})$ (see e.g. [36, p.33]). In fact $p$ is a nonnegative function with $\int_{0}^{\bar{\theta}} p(x) d x>0$. Subsequently, we further determine $p \geq 0$ on $[\bar{\theta}, 1]$ by (30). The solution $p$ on $[0,1]$ depends linearly on $\pi_{0}$ and so there exists a unique $\pi_{0}$ such that $\int_{0}^{1} p(x) d x+\pi_{0}=1$. After we uniquely solve $p$ for 30 , we integrate both sides of this equation on $[0,1]$ and obtain $\int_{0}^{1} p(x) d x=\int_{0}^{\bar{\theta}} p(x) d x+\pi_{0}$, which implies that (29) is satisfied.

\subsection{Special Case}

Now we suppose $Q_{0}(d y \mid x)$ has uniform distribution on $[x, 1]$ for all fixed $0 \leq x<1$, and $R(x, z)=R_{1}(x) R_{2}(z)=x(c+z)$, where $R_{1}(x)=x, R_{2}(z)=c+z$ and $c>0$. In this case, (A2)-(A6) are satisfied. For (21), we have

$$
\bar{v}(x)= \begin{cases}\frac{\beta}{1-x} \int_{x}^{1} \bar{v}(y) d y+R_{1}(x) R_{2}(\bar{z}), & 0 \leq x \leq \bar{\theta}, \\ \beta \bar{v}(0)+R_{1}(x) R_{2}(\bar{z})+\bar{\gamma}, & \bar{\theta} \leq x \leq 1 .\end{cases}
$$

Denote $\varphi(x)=\int_{x}^{1} \bar{v}(y) d y$. Then

$$
\dot{\varphi}(x)=-\frac{\beta}{1-x} \varphi-R_{1}(x) R_{2}(\bar{z}), \quad 0 \leq x \leq \bar{\theta} .
$$

Taking the initial condition $\varphi(0)$, we have

$$
\varphi(x)=\varphi(0)(1-x)^{\beta}-(1-x)^{\beta} \int_{0}^{x} \frac{R_{1}(\tau) R_{2}(\bar{z})}{(1-\tau)^{\beta}} d \tau .
$$

On $[0, \bar{\theta}]$,

$$
\begin{aligned}
\bar{v}(x) & =(1-x)^{\beta-1} \bar{v}(0)-\beta(1-x)^{\beta-1} \int_{0}^{x} \frac{R_{1}(\tau) R_{2}(\bar{z})}{(1-\tau)^{\beta}} d \tau+R_{1}(x) R_{2}(\bar{z}) \\
& =(1-x)^{\beta-1}\left[\bar{v}(0)-\frac{\beta(c+\bar{z})}{(1-\beta)(2-\beta)}\right]+(c+\bar{z})\left[\frac{\beta}{(1-\beta)(2-\beta)}+\frac{2 x}{2-\beta}\right] .
\end{aligned}
$$

By the continuity of $\bar{v}$ and its form on $[\bar{\theta}, 1]$, we have

$$
\bar{v}(\bar{\theta})=\beta \bar{v}(0)+\bar{\theta}(\bar{z}+c)+\bar{\gamma}
$$

Hence,

$$
\left[(1-\bar{\theta})^{\beta-1}-\beta\right] \bar{v}(0)=\frac{\beta(c+\bar{z})\left[(1-\bar{\theta})^{\beta-1}-1\right]}{(1-\beta)(2-\beta)}-\frac{\beta(c+\bar{z}) \bar{\theta}}{2-\beta}+\bar{\gamma} .
$$

On the other hand, since $\bar{v}$ is increasing and $\bar{\theta}$ is the threshold, we have 


$$
\begin{aligned}
\bar{v}(\bar{\theta}) & =\beta \int_{\bar{\theta}}^{1}[\beta \bar{v}(0)+(c+z) y+\bar{\gamma}] \frac{1}{1-\bar{\theta}} d y+(c+\bar{z}) \bar{\theta} \\
& =\beta^{2} \bar{v}(0)+\beta \bar{\gamma}+\frac{\beta(c+\bar{z})}{2}+\left(\frac{\beta}{2}+1\right)(c+\bar{z}) \bar{\theta},
\end{aligned}
$$

which combined with $(33)$ gives

$$
\frac{\beta}{2}(c+\bar{z})(1+\bar{\theta})=(\beta \bar{v}(0)+\bar{\gamma})(1-\beta) .
$$

Given the special form of $Q_{0}(d y \mid x)$, (26) becomes

$$
w(x)= \begin{cases}\frac{\beta}{1-x} \int_{x}^{1} w(y) d y+R_{1}(x) R_{2}^{\prime}(\bar{z}) z_{\gamma}, & 0 \leq x \leq \bar{\theta}, \\ \beta w(0)+R_{1}(x) R_{2}^{\prime}(\bar{z}) z_{\gamma}+1, & \bar{\theta}<x \leq 1 .\end{cases}
$$

The computation of $w$ now reduces to uniquely solving $w(0)$. By the expression of $w$ on $[0, \bar{\theta}]$, we have

$$
\begin{aligned}
w(\bar{\theta}) & =\beta \int_{\bar{\theta}}^{1} w(y) Q_{0}(d y \mid \bar{\theta})+R_{1}(\bar{\theta}) R_{2}^{\prime}(\bar{z}) z_{\gamma} \\
& =\beta^{2} w(0)+\beta+R_{1}(\bar{\theta}) R_{2}^{\prime}(\bar{z}) z_{\gamma}+\frac{\beta R_{2}^{\prime}(\bar{z}) z_{\gamma}}{1-\bar{\theta}} \int_{\bar{\theta}}^{1} R_{1}(y) d y \\
& =\beta^{2} w(0)+\beta+\bar{\theta} z_{\gamma}+\beta z_{\gamma} \frac{1+\bar{\theta}}{2} .
\end{aligned}
$$

For $x \in[0, \bar{\theta}]$, we further write

$$
w(x)=\frac{\beta}{1-x} \int_{x}^{1} w(y) d y+R_{1}(x) R_{2}^{\prime}(\bar{z}) z_{\gamma},
$$

and solve

$$
w(x)=(1-x)^{\beta-1} w(0)+z_{\gamma} x-\beta z_{\gamma}\left[\frac{(1-x)^{\beta-1}}{(1-\beta)(2-\beta)}-\frac{1}{1-\beta}+\frac{1-x}{2-\beta}\right],
$$

which further gives

$$
w(\bar{\theta})=(1-\bar{\theta})^{\beta-1} w(0)+z_{\gamma} \bar{\theta}-\beta z_{\gamma}\left[\frac{(1-\bar{\theta})^{\beta-1}}{(1-\beta)(2-\beta)}-\frac{1}{1-\beta}+\frac{1-\bar{\theta}}{2-\beta}\right] .
$$

By (37)-(38), we have

$$
\left[\beta^{-1}(1-\bar{\theta})^{\beta-1}-\beta\right] w(0)=1+z_{\gamma}\left(\frac{1+\bar{\theta}}{2}+\frac{(1-\bar{\theta})^{\beta-1}}{(1-\beta)(2-\beta)}+\frac{1-\bar{\theta}}{2-\beta}-\frac{1}{1-\beta}\right) .
$$

Now from (30) we have 


$$
p(x)= \begin{cases}\int_{0}^{x} \frac{1}{1-y} p(y) d y+\pi_{0}, & 0 \leq x<\bar{\theta}, \\ \int_{0}^{\bar{\theta}} \frac{1}{1-y} p(y) d y+\pi_{0}, & \bar{\theta} \leq x \leq 1,\end{cases}
$$

which determines

$$
p(x)= \begin{cases}\frac{\pi_{0}}{1 \bar{\pi}_{0}^{x}}, & 0 \leq x<\bar{\theta} \\ \frac{1-\bar{\theta}}{1-\bar{\theta}} \leq x \leq 1\end{cases}
$$

where $\pi_{0}=\frac{1}{2-\ln (1-\bar{\theta})}$. We determine the mean field

$$
\bar{z}=\int_{0}^{\bar{\theta}} x p(x) d x+\int_{\bar{\theta}}^{1} x p(x) d x=\pi_{0}\left(\frac{1-\bar{\theta}}{2}-\ln (1-\bar{\theta})\right) .
$$

We further obtain $\frac{d z}{d \gamma}$ at $\bar{\gamma}$ as

$$
z_{\gamma}=\frac{\ln (1-\bar{\theta})-3+\frac{4}{1-\theta}}{2[2-\ln (1-\bar{\theta})]^{2}} \theta_{\gamma} .
$$

We note that a perturbation analysis directly based on the general case (30) is more complicated.

Now (28) reduces to

$$
\left[\frac{\beta}{1-\bar{\theta}} \int_{\bar{\theta}}^{1} \frac{\bar{v}(y)}{1-\bar{\theta}} d y-\frac{\beta \bar{v}(\bar{\theta})}{1-\bar{\theta}}\right] \theta_{\gamma}=1+\beta w(0)-\beta \int_{\bar{\theta}}^{1} \frac{w(y)}{1-\bar{\theta}} d y .
$$

By the expression of $\bar{v}$ in (32) and $w$ in (36) at $\theta=\bar{\theta}$, we obtain

$$
\frac{(1-\beta) \bar{v}(\bar{\theta})-\bar{\theta}(c+\bar{z})}{1-\bar{\theta}} \theta_{\gamma}=1+\beta w(0)-w(\bar{\theta})+\bar{\theta} z_{\gamma}
$$

Recalling (33) and (37), we have

$$
\frac{(1-\beta)[\beta \bar{v}(0)+\bar{\gamma}]-\beta \bar{\theta}(\bar{z}+c)}{1-\bar{\theta}} \theta_{\gamma}-\beta(1-\beta) w(0)+\frac{1+\bar{\theta}}{2} \beta z_{\gamma}=1-\beta .
$$

By combining (34), (35) and (40), we have

$$
\begin{aligned}
& \bar{v}(0)=\left[(1-\bar{\theta})^{\beta-1}-\beta\right]^{-1}\left[\frac{\beta(c+\bar{z})\left[(1-\bar{\theta})^{\beta-1}-1\right]}{(1-\beta)(2-\beta)}-\frac{\beta(c+\bar{z}) \bar{\theta}}{2-\beta}+\bar{\gamma}\right], \\
& \bar{\theta}=\frac{2(1-\beta)(\beta \bar{v}(0)+\bar{\gamma})}{\beta(c+\bar{z})}-1, \\
& \bar{z}=\frac{1}{2-\ln (1-\bar{\theta})}\left(\frac{1-\bar{\theta}}{2}-\ln (1-\bar{\theta})\right) .
\end{aligned}
$$




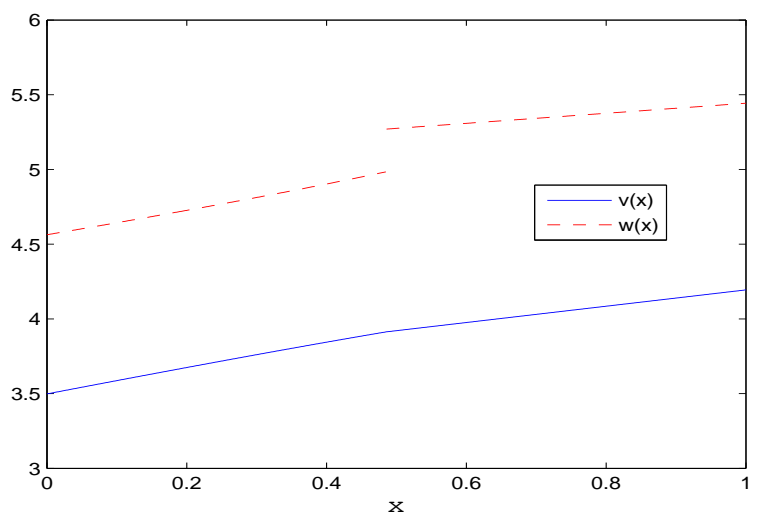

Fig. 1 Value function $v$ and perturbation function $w$

Next, combining (39), (41) and (42), we obtain

$$
\begin{aligned}
& \frac{(1-\beta)[\beta \bar{v}(0)+\bar{\gamma}]-\beta \bar{\theta}(\bar{z}+c)}{1-\bar{\theta}} \theta_{\gamma}-\beta(1-\beta) w(0)+\frac{1+\bar{\theta}}{2} \beta z_{\gamma}=1-\beta \\
& {\left[\beta^{-1}(1-\bar{\theta})^{\beta-1}-\beta\right] w(0)=1+z_{\gamma}\left(\frac{1+\bar{\theta}}{2}+\frac{(1-\bar{\theta})^{\beta-1}}{(1-\beta)(2-\beta)}+\frac{1-\bar{\theta}}{2-\beta}-\frac{1}{1-\beta}\right)} \\
& z_{\gamma}=\frac{\ln (1-\bar{\theta})-3+\frac{4}{1-\bar{\theta}}}{2[2-\ln (1-\bar{\theta})]^{2}} \theta_{\gamma}
\end{aligned}
$$

After $(\bar{v}(0), \bar{z}, \bar{\theta})$ has been determined from (43)-(45), the above gives a linear equation system with unknowns $w(0), \theta_{\gamma}$ and $z_{\gamma}$.

Example 2. We take $R_{1}(x)=x$ and $R_{2}(z)=0.2+z, \bar{\gamma}=0.5, \beta=0.92$ We numerically solve (43)-(45) to obtain $\bar{v}(0)=3.497854, \bar{\theta}=0.485162, \bar{z}=0.345854$, and (46)-(48) to obtain $w(0)=4.563055, \theta_{\gamma}=1.162861, z_{\gamma}=0.336380$. The curves of $v(x)$ and $w(x)$ are displayed in Fig. 1, where $w$ has a discontinuity at $x=\bar{\theta}$ as discussed in Remark 2. The positive value of $\theta_{\gamma}$ implies the threshold increases with $\gamma$, as asserted in Theorem 3 .

\section{Conclusion}

This paper considers mean field games in a framework of binary Markov decision processes (MDP) and establishes existence and uniqueness of stationary equilib-

${ }^{2}$ Corrected on Oct 10, 2020 by adding the value of $\beta$ and correcting the parameter in $R_{2}(z)$. 
ria. The resulting policy has a threshold structure. We further analyze comparative statics to address the impact of parameter variations in the model.

For future research, there are some potentially interesting extensions. One may consider a heterogenous population and study the emergence of free-riders who care more about their own effort costs and have less incentive to contribute to the common benefit of the population. Another modelling of a quite different nature involves negative externalities where other players' improvement brings more pressure on the player in question. For instance, this arises in competitions for market share. The modelling and analysis of the agent behavior will be of interest.

\section{Appendix A: Preliminaries on Ergodicity}

Assume (A3). The next two lemmas determine the limiting distribution of the state process under threshold policies.

Lemma A.1. $i$ ) If $\theta=0$, then the distribution of $x_{t}^{i}$ remains to be the dirac measure $\delta_{0}$ for all $t \geq 1$, for any $x_{0}^{i}$.

ii) If $\theta=1$ or $\theta=1^{+}$, the distribution of $x_{t}^{i}$ converges to the dirac measure $\delta_{1}$ weakly.

Proof. Part i) is obvious and part ii) follows from (A3).

Let $x_{t}^{i, \theta}$ denote the state process generated by the $\theta$-threshold policy with $\theta \in$ $(0,1)$, and let $P_{\theta}^{t}(x, \cdot)$ be the distribution of $x_{t}^{i, \theta}$ given $x_{0}^{i, \theta}=x$.

Lemma A.2. For $\theta \in(0,1),\left\{x_{t}^{i, \theta}, t \geq 0\right\}$ is uniformly ergodic with stationary probability distribution $\pi_{\theta}$, i.e.,

$$
\sup _{x \in \mathbf{S}}\left\|P_{\theta}^{t}(x, \cdot)-\pi_{\theta}\right\|_{\mathrm{TV}} \leq K r^{t},
$$

for some constants $K>0$ and $r \in(0,1)$, where $\|\cdot\|_{\mathrm{TV}}$ is the total variation norm of signed measures.

Proof. The proof is similar to that of the ergodicity theorem in [27], which assumed $\left(\mathrm{A} 3^{\prime}\right)$. We use (A3)-iii) to estimate $r$.

We take $C_{s}=\{0\}$ as a small set and $\theta \in(0,1)$. The $\theta$-threshold policy gives

$$
P\left(x_{2}^{i, \theta}=0 \mid x_{0}^{i, \theta}=0\right) \geq \int_{\theta}^{1} q(y \mid 0) d y=: \varepsilon_{0} .
$$

So for any Borel set $B, P\left(x_{2}^{i, \theta} \in B \mid x_{0}^{i, \theta}=0\right) \geq \varepsilon_{0} \delta_{0}(B)$, where $\delta_{0}$ is the dirac measure. For $\theta^{\prime}$ in a small neighborhood of $\theta$, we can ensure that the $\theta^{\prime}$-threshold policy gives

$$
P\left(x_{2}^{i, \theta^{\prime}} \in B \mid x_{0}^{i, \theta^{\prime}}=0\right) \geq \frac{\varepsilon_{0}}{2} \delta_{0}(B) .
$$


Lemma A.3. Suppose $\theta, \theta^{\prime} \in(0,1)$ for two threshold policies. Let the corresponding stationary distributions of the state process by $\pi$ and $\pi^{\prime}$. Then

$$
\lim _{\theta^{\prime} \rightarrow \theta}\left\|\pi^{\prime}-\pi\right\|_{\mathrm{TV}}=0 .
$$

Proof. Fix $\theta \in(0,1)$. By (A.3) and [41], there exist a neighborhood $I_{0}=(\theta-$ $\left.\kappa_{0}, \theta+\kappa_{0}\right) \subset(0,1)$ and two constants $C, r \in(0,1)$ such that for all $\theta^{\prime} \in I_{0}$,

$$
\left\|P_{\theta}^{t}(x, \cdot)-\pi\right\|_{\mathrm{TV}} \leq C r^{t}, \quad\left\|P_{\theta^{\prime}}^{t}(x, \cdot)-\pi^{\prime}\right\|_{\mathrm{TV}} \leq C r^{t}, \quad \forall x \in[0,1] .
$$

Subsequently,

$$
\left\|\pi^{\prime}-\pi\right\|_{\mathrm{TV}} \leq\left\|P_{\theta^{\prime}}^{t}(0, \cdot)-P_{\theta}^{t}(0, \cdot)\right\|_{\mathrm{TV}}+2 C r^{t} .
$$

For any given $\varepsilon>0$, fix a large $k_{0}$ such that $2 C r^{k_{0}} \leq \varepsilon / 2$. We show for all $\theta^{\prime}$ sufficiently close to $\theta$,

$$
\left\|P_{\theta^{\prime}}^{k_{0}}(0, \cdot)-P_{\theta}^{k_{0}}(0, \cdot)\right\|_{\mathrm{TV}} \leq \varepsilon / 2 .
$$

Given two probability measures $\mu_{t}, \mu_{t}^{\prime}$, define the probability measures $\mu_{t+1}$ and $\mu_{t+1}^{\prime}$,

$$
\mu_{t+1}(B)=\int_{\mathbf{S}} P_{\theta}(y, B) \mu_{t}(d y), \quad \mu_{t+1}^{\prime}(B)=\int_{\mathbf{S}} P_{\theta^{\prime}}(y, B) \mu_{t}^{\prime}(d y),
$$

for Borel set $B \subset[0,1]$. Then

$$
\begin{aligned}
\left|\mu_{t+1}(B)-\mu_{t+1}^{\prime}(B)\right| \leq & \left|\int_{\mathbf{S}} P_{\theta}(y, B) \mu_{t}(d y)-\int_{\mathbf{S}} P_{\theta^{\prime}}(y, B) \mu_{t}(d y)\right| \\
& +\left|\int_{\mathbf{S}} P_{\theta^{\prime}}(y, B) \mu_{t}(d y)-\int_{\mathbf{S}} P_{\theta^{\prime}}(y, B) \mu_{t}^{\prime}(d y)\right| \\
& =: D_{1}+D_{2} .
\end{aligned}
$$

We have

$$
D_{2}=\left|\int_{\mathbf{S}} P_{\theta^{\prime}}(y, B) \mu_{t}(d y)-\int_{\mathbf{S}} P_{\theta^{\prime}}(y, B) \mu_{t}^{\prime}(d y)\right| \leq 2\left\|\mu_{t}-\mu_{t}^{\prime}\right\|_{\mathrm{TV}}
$$

Denote $\underline{\theta}=\min \left\{\theta, \theta^{\prime}\right\}$ and $\bar{\theta}=\max \left\{\theta, \theta^{\prime}\right\}$. Then

$$
D_{1}=\left|-\int_{[\underline{\theta}, \bar{\theta})} Q_{0}(B \mid y) \mu_{t}(d y)+1_{B}(0) \mu_{t}([\underline{\theta}, \bar{\theta}))\right| \leq \mu_{t}([\underline{\theta}, \bar{\theta})) .
$$

Setting $\mu_{0}=\mu_{0}^{\prime}=\delta_{0}$, then $\mu_{t}=P_{\theta}^{t}(0, \cdot), \mu_{t}^{\prime}=P_{\theta^{\prime}}^{t}(0, \cdot)$. Hence,

$$
\left|P_{\theta^{\prime}}^{t+1}(0, B)-P_{\theta}^{t+1}(0, B)\right| \leq 2\left\|P_{\theta^{\prime}}^{t}(0, \cdot)-P_{\theta}^{t}(0, \cdot)\right\|_{\mathrm{TV}}+P_{\theta}^{t}(0,[\underline{\theta}, \bar{\theta})),
$$

which implies

$$
\left\|P_{\theta^{\prime}}^{t+1}(0, \cdot)-P_{\theta}^{t+1}(0, \cdot)\right\|_{\mathrm{TV}} \leq 4\left\|P_{\theta^{\prime}}^{t}(0, \cdot)-P_{\theta}^{t}(0, \cdot)\right\|_{\mathrm{TV}}+2 P_{\theta}^{t}\left(0,\left[\theta, \theta^{\prime}\right)\right) .
$$


For $\mu_{0}=\mu_{0}^{\prime}=\delta_{0}$, we have $P_{\theta}^{1}(0, \cdot)=P_{\theta^{\prime}}^{1}(0, \cdot)$. It is clear from A.5) and Lemma4 that for each $t \geq 1$,

$$
\lim _{\theta^{\prime} \rightarrow \theta}\left\|P_{\theta^{\prime}}^{t}(0, \cdot)-P_{\theta}^{t}(0, \cdot)\right\|_{\mathrm{TV}}=0, \quad \lim _{\theta^{\prime} \rightarrow \theta} P_{\theta}^{t}(0,[\underline{\theta}, \bar{\theta}))=0 .
$$

Therefore, for the fixed $k_{0}$, there exists $\delta>0$ such that for all $\theta^{\prime}$ satisfying $\left|\theta^{\prime}-\theta\right|<$ $\delta,\left\|P_{\theta^{\prime}}^{k_{0}}(0, \cdot)-P_{\theta}^{k_{0}}(0, \cdot)\right\|_{\mathrm{TV}}<\frac{\varepsilon}{2}$ and $\left\|\pi^{\prime}-\pi\right\|_{\mathrm{TV}} \leq \varepsilon$. The lemma follows.

\section{Appendix B: Cycle Average of A Regenerative Process}

Let $0<r<r^{\prime}<1$. Consider a Markov process $\left\{Y_{t}, t \geq 0\right\}$ with state space $[0,1]$ and transition kernel $Q_{Y}(\cdot \mid y)$ which satisfies $Q_{Y}([y, 1] \mid y)=1$ for any $y \in[0,1]$ and is stochastically increasing. Suppose $Y_{0} \equiv y_{0}<r$. Define the stopping times

$$
\tau=\inf \left\{t \mid Y_{t} \geq r\right\}, \quad \tau^{\prime}=\inf \left\{t \mid Y_{t} \geq r^{\prime}\right\} .
$$

Lemma B.1. If $E \tau<\infty$, then $E \sum_{t=0}^{\tau} Y_{t}<\infty$ and

$$
\frac{E \sum_{t=0}^{\tau} Y_{t}}{1+E \tau}=\frac{E Y_{0}+E Y_{1}+\sum_{k=1}^{\infty} E\left(Y_{k+1} 1_{\left\{Y_{k}<r\right\}}\right)}{2+\sum_{k=1}^{\infty} P\left(Y_{k}<r\right)} .
$$

Proof. Since $0 \leq Y_{t} \leq 1$ w.p. $1, E \sum_{t=0}^{\tau} Y_{t} \leq 1+E \tau$. It is clear that $\{\tau \geq k\}=\left\{Y_{k-1}<\right.$ $r$ f for $k \geq 1$. We have

$$
E \tau=\sum_{k=1}^{\infty} P(\tau \geq k)=1+\sum_{k=1}^{\infty} P\left(Y_{k}<r\right)
$$

and

$$
\begin{aligned}
E \sum_{t=0}^{\tau} Y_{t} & =E \sum_{k=1}^{\infty}\left(\sum_{t=0}^{k} Y_{t}\right) 1_{\{\tau=k\}} \\
& =E Y_{0}+E Y_{1}+\sum_{k=2}^{\infty} E\left(Y_{k} 1_{\{\tau \geq k\}}\right) \\
& =E Y_{0}+E Y_{1}+\sum_{k=1}^{\infty} E\left(Y_{k+1} 1_{\left\{Y_{k}<r\right\}}\right) .
\end{aligned}
$$

The lemma follows.

Lemma B.2. Assume $E \tau^{\prime}<\infty$. We have

$$
\frac{E \sum_{t=0}^{\tau} Y_{t}}{1+E \tau} \leq \frac{E \sum_{t=0}^{\tau^{\prime}} Y_{t}}{1+E \tau^{\prime}}
$$

Proof. $E \tau<\infty$ since $\tau \leq \tau^{\prime}$ w.p.1. For $k \geq 1$, denote 


$$
\begin{aligned}
& p_{k}=P\left(Y_{k}<r\right), \quad \eta_{k}=P\left(r \leq Y_{k}<r^{\prime}\right), \\
& m_{k}=E\left(Y_{k+1} 1_{\left\{Y_{k}<r\right\}}\right), \quad \Delta_{k}=E\left(Y_{k+1} 1_{\left\{r \leq Y_{k}<r^{\prime}\right\}}\right) .
\end{aligned}
$$

By Lemma B.1

$$
\begin{aligned}
\frac{E \sum_{t=0}^{\tau} Y_{t}}{1+E \tau} & =\frac{E Y_{0}+E Y_{1}+\sum_{k=1}^{\infty} m_{k}}{2+\sum_{k=1}^{\infty} p_{k}} \\
\frac{E \sum_{t=0}^{\tau^{\prime}} Y_{t}}{1+E \tau^{\prime}} & =\frac{E Y_{0}+E Y_{1}+\sum_{k=1}^{\infty}\left(m_{k}+\Delta_{k}\right)}{2+\sum_{k=1}^{\infty}\left(p_{k}+\eta_{k}\right)} .
\end{aligned}
$$

So $\mathrm{B} .3$ is equivalent to

$$
\left(E Y_{0}+E Y_{1}+\sum_{k=1}^{\infty} m_{k}\right)\left(\sum_{k=1}^{\infty} \eta_{k}\right) \leq\left(\sum_{k=1}^{\infty} \Delta_{k}\right)\left(2+\sum_{k=1}^{\infty} p_{k}\right)
$$

By the stochastic monotonicity of $Q_{Y}$, we have

$$
\begin{aligned}
E\left[Y_{k+1} 1_{\left\{Y_{k}<r\right\}} \mid Y_{k}\right] & =1_{\left\{Y_{k}<r\right\}} \int_{0}^{1} y Q_{Y}\left(d y \mid Y_{k}\right) \\
& \leq 1_{\left\{Y_{k}<r\right\}} \int_{0}^{1} y Q_{Y}(d y \mid r)=: c_{r} 1_{\left\{Y_{k}<r\right\}} .
\end{aligned}
$$

Note that

$$
c_{r}=\int_{y \geq r} y Q_{Y}(d y \mid r) \geq r
$$

Moreover,

$$
\begin{aligned}
E\left[Y_{k+1} 1_{\left\{r \leq Y_{k}<r^{\prime}\right\}} \mid Y_{k}\right] & =1_{\left\{r \leq Y_{k}<r^{\prime}\right\}} \int_{0}^{1} y Q_{Y}\left(d y \mid Y_{k}\right) \\
& \geq c_{r} 1_{\left\{r \leq Y_{k}<r^{\prime}\right\}}
\end{aligned}
$$

It follows that

$$
m_{k}=E\left[Y_{k+1} 1_{\left\{Y_{k}<r\right\}}\right] \leq c_{r} p_{k}, \quad \Delta_{k}=E\left[Y_{k+1} 1_{\left\{r \leq Y_{k}<r^{\prime}\right\}}\right] \geq c_{r} \eta_{k} .
$$

Since $Y_{0}=y_{0}<r$,

$$
E\left[Y_{1} \mid Y_{0}\right]=\int_{0}^{1} y Q_{Y}\left(d y \mid Y_{0}\right) \leq c_{r}
$$

Hence, $E\left(Y_{0}+Y_{1}\right) \leq r+c_{r}$. By (B.6) and (B.5), 


$$
\begin{aligned}
& \left(E Y_{0}+E Y_{1}+\sum_{k=1}^{\infty} m_{k}\right)\left(\sum_{k=1}^{\infty} \eta_{k}\right)-\left(\sum_{k=1}^{\infty} \Delta_{k}\right)\left(2+\sum_{k=1}^{\infty} p_{k}\right) \\
\leq & \left(r+c_{r}+c_{r} \sum_{k=1}^{\infty} p_{k}\right)\left(\sum_{k=1}^{\infty} \eta_{k}\right)-c_{r}\left(\sum_{k=1}^{\infty} \eta_{k}\right)\left(2+\sum_{k=1}^{\infty} p_{k}\right) \\
= & \left(r-c_{r}\right) \sum_{k=1}^{\infty} \eta_{k} \leq 0,
\end{aligned}
$$

which establishes (B.4).

Remark B.1. If for each $y \in[0,1), Q_{Y}(d x \mid y)$ has probability density function $q_{Y}(x \mid y)>$ 0 for $x \in(y, 1)$, then $c_{r}>r$ and $\eta_{k}>0$ for all $k \geq 1$. In this case, a strict inequality holds for (B.3).

\section{Appendix C}

We assume (A3). Let $\left\{x_{t}^{i, \theta}, t \geq 0\right\}$ be the Markov chain generated by a $\theta$-threshold policy with $0<\theta<1$, where $x_{0}^{i, \theta}$ is given. By LemmaA.2 $\left\{x_{t}^{i, \theta}, t \geq 0\right\}$ is ergodic. We next define an auxiliary Markov chain $\left\{Y_{t}, t \geq 0\right\}$ with $Y_{0}=0$ and the same transition kernel as $x_{t}^{i, \theta}$. Denote $S_{t}=\sum_{i=0}^{t} Y_{i}$ for $t \geq 0$. Define $\tau=\inf \left\{t \mid Y_{t} \geq \theta\right\}$.

Lemma C.1. We have

$$
\lim _{k \rightarrow \infty} \frac{1}{k} \sum_{t=0}^{k-1} Y_{t}=\frac{E S_{\tau}}{1+E \tau} \quad \text { w.p. } 1 .
$$

Proof. By (A3), we can show $E \tau<\infty$. Since $\left\{Y_{t}, t \geq 0\right\}$ has the same transition probability kernel as $\left\{x_{t}^{i, \theta}, t \geq 0\right\}$, it is ergodic, and therefore the left hand side of (C.1) has a constant limit w.p.1. Define $T_{0}=0$ and $T_{n}$ as the time for $\left\{Y_{t}, t \geq 0\right\}$ to return to state 0 for the $n$th time. So $T_{1}=\tau+1$. Define $B_{n}=\sum_{t=T_{n-1}}^{T_{n}-1} Y_{t}$ for $n \geq 1$. We observe that $\left\{Y_{t}, t \geq 0\right\}$ is a regenerative process (see e.g. [6, 51] and [7, Theorem 4]) with regeneration times $\left\{T_{n}, n \geq 1\right\}$ and that $\left\{B_{n}, n \geq 1\right\}$ is a sequence of i.i.d. random variables. Note that $B_{1}=S_{\tau}$ is the sum of $\tau+1$ terms. By the strong law of large numbers for regenerative processes [6, pp. 177], the lemma follows.

Suppose $0<\theta<\theta^{\prime}<1$. Then there exist two constants $C_{\theta}, C_{\theta^{\prime}}$ such that

$$
\lim _{k \rightarrow \infty} \frac{1}{k} \sum_{t=0}^{k-1} x_{t}^{i, \theta}=C_{\theta}, \quad \lim _{k \rightarrow \infty} \frac{1}{k} \sum_{t=0}^{k-1} x_{t}^{i, \theta^{\prime}}=C_{\theta^{\prime}}, \quad \text { w.p.1. }
$$

Lemma C.2. We have $C_{\theta} \leq C_{\theta^{\prime}}$.

Proof. Due to the ergodicity of the Markov chain, $C_{\theta}$ (resp., $C_{\theta^{\prime}}$ ) does not depend on $x_{0}^{i, \theta}$ (resp., $x_{0}^{i, \theta^{\prime}}$ ). Therefore, $\lim _{k \rightarrow \infty} \frac{1}{k} \sum_{t=0}^{k-1} Y_{t}=C_{\theta}$ w.p.1. The lemma follows from Lemmas C.1 and B.2. 


\section{Appendix D: An Auxiliary MDP}

Assume (A3). This appendix introduces an auxiliary control problem to show the effect of the effort cost on the threshold parameter of the optimal policy. The state and control processes $\left\{\left(x_{t}^{i}, a_{t}^{i}\right), t \geq 0\right\}$ are specified by (1)-(2). The cost has the form

$$
J_{i}^{r}=E \sum_{t=0}^{\infty} \rho^{t}\left(R_{1}\left(x_{t}^{i}\right)+r 1_{\left\{a_{t}^{i}=a_{1}\right\}}\right)
$$

where $R_{1}$ is continuous and strictly increasing on $[0,1]$ and $\rho \in(0,1), r \in(0, \infty)$. Let $r$ take two different values $0<\gamma_{1}<\gamma_{2}$ and write the corresponding dynamic programming equation

$v_{l}(x)=\min \left\{\rho \int_{0}^{1} v_{l}(y) Q_{0}(d y \mid x)+R_{1}(x), \quad \rho v_{l}(0)+R_{1}(x)+\gamma_{l}\right\}, \quad l=1,2, x \in \mathbf{S}$.

By the method in proving Lemma 1, it can be shown that there exists a unique solution $v_{l} \in C([0,1], \mathbb{R})$ and that the optimal policy $a^{i, l}(x)$ is a threshold policy. If $\rho \int_{0}^{1} v_{l}(y) Q_{0}(d y \mid 1)<\rho v_{l}(0)+\gamma_{l}, a^{i, l}(x) \equiv a_{0}$, and we follow the notation in Section 3 to denote the threshold $\theta_{l}=1^{+}$. Otherwise, $a^{i, l}(x)$ is a $\theta_{l}$-threshold policy with $\theta_{l} \in[0,1]$, i.e., $a^{i, l}(x)=a_{1}$ if $x \geq \theta_{l}$, and $a^{i, l}(x)=a_{0}$ if $x<\theta_{l}$.

Lemma D.1. If $\theta_{1} \in(0,1), \theta_{2} \neq \theta_{1}$.

Proof. We prove by contradiction. Suppose for some $\theta \in(0,1)$,

$$
\theta_{1}=\theta_{2}=\theta
$$

Under (D.3), the resulting optimal policy leads to the representation (see e.g. [23, pp. 22])

$$
v_{l}(x)=E \sum_{t=0}^{\infty} \rho^{t}\left[R_{1}\left(x_{t}^{i}\right)+\gamma_{l} 1_{\left\{a_{t}^{i}=a_{1}\right\}}\right], \quad l=1,2,
$$

where $\left\{x_{t}^{i}, t \geq 0\right\}$ is generated by the $\theta$-threshold policy $a_{t}^{i}\left(x_{t}^{i}\right)$ and $x_{0}^{i}=x$. Denote $\delta_{21}=\gamma_{2}-\gamma_{1}$.

For fixed $x \geq \theta$ and $x_{0}^{i}=x$, denote the resulting optimal state and control processes by $\left\{\left(\hat{x}_{t}^{i}, \hat{a}_{t}^{i}\right), t \geq 0\right\}$. Then $\hat{a}_{0}^{i}=a_{1}$ w.p.1., and

$$
v_{2}(x)-v_{1}(x)=\delta_{21}+\delta_{21} E \sum_{t=1}^{\infty} \rho^{t} 1_{\left\{\hat{a}_{t}^{i}=a_{1}\right\}}, \quad x \geq \theta .
$$

Next consider $x_{0}^{i}=0$ and denote the optimal state and control processes by $\left\{\left(\check{x}_{t}^{i}, \check{a}_{t}^{i}\right), t \geq 0\right\}$. Then

$$
v_{2}(0)-v_{1}(0)=\delta_{21} E \sum_{t=0}^{\infty} \rho^{t} 1_{\left\{\check{a}_{t}^{i}=a_{1}\right\}}=: \Delta .
$$


It is clear that $\hat{x}_{1}^{i}=0$ w.p. 1 . By the optimality principle, $\left\{\left(\hat{x}_{t}^{i}, \hat{a}_{t}^{i}\right), t \geq 1\right\}$ may be interpreted as the optimal state and control processes of the MDP with initial state 0 at $t=1$. Hence the two processes $\left\{\left(\hat{x}_{t}^{i}, \hat{a}_{t}^{i}\right), t \geq 1\right\}$ and $\left\{\left(\check{x}_{t}^{i}, \breve{a}_{t}^{i}\right), t \geq 0\right\}$, where $\check{x}_{0}^{i}=0$, have the same finite dimensional distributions. In particular, $\hat{a}_{t+1}^{i}$ and $\breve{a}_{t}^{i}$ have the same distribution for $t \geq 0$. Therefore,

$$
E \sum_{t=1}^{\infty} \rho^{t-1} 1_{\left\{\hat{a}_{t}^{i}=a_{1}\right\}}=E \sum_{t=0}^{\infty} \rho^{t} 1_{\left\{\check{a}_{t}^{i}=a_{1}\right\}} .
$$

It follows that

$$
v_{2}(x)-v_{1}(x)=\delta_{21}+\rho \Delta, \quad \forall x \geq \theta .
$$

Combining (D.2) and (D.3) gives

$$
\rho \int_{0}^{1} v_{l}(y) Q_{0}(d y \mid \theta)=\rho v_{l}(0)+\gamma_{l}, \quad l=1,2
$$

which implies

$$
\rho \int_{0}^{1}\left[v_{2}(x)-v_{1}(x)\right] Q_{0}(d x \mid \theta)=\delta_{21}+\rho \Delta .
$$

By $Q_{0}([0, \theta) \mid \theta)=0$ and (D.4), (D.5) further yields $\rho\left(\delta_{21}+\rho \Delta\right)=\delta_{21}+\rho \Delta$, which is impossible since $0<\rho<1$ and $\delta_{21}+\rho \Delta>0$. Therefore, (D.3) does not hold. This completes the proof.

For the MDP with cost (D.1), we continue to analyze the dynamic programming equation

$$
v_{r}(x)=\min \left[\rho \int_{0}^{1} v_{r}(y) Q_{0}(d y \mid x)+R_{1}(x), \quad \rho v_{r}(0)+R_{1}(x)+r\right] .
$$

For each fixed $r \in(0, \infty)$, we obtain the optimal policy as a threshold policy with threshold parameter $\theta(r)$. By evaluating the cost (D.1) associated with the two policies $a_{t}^{i}\left(x_{t}^{i}\right) \equiv a_{0}$ and $a_{t}^{i}\left(x_{t}^{i}\right) \equiv a_{1}$, respectively, we have the prior estimate

$$
v_{r}(x) \leq \min \left\{\frac{R_{1}(1)}{1-\rho}, R_{1}(x)+\frac{r+\rho R_{1}(0)}{1-\rho}\right\} .
$$

On the other hand, let $\left\{x_{t}^{i}, t \geq 0\right\}$ with $x_{0}^{i}=x$ be generated by any fixed Markov policy. Then

$$
E \sum_{t=0}^{\infty} \rho^{t}\left(R_{1}\left(x_{t}^{i}\right)+r 1_{\left\{a_{t}^{i}=a_{1}\right\}}\right) \geq R_{1}(x)+\sum_{t=1}^{\infty} \rho^{t} R_{1}(0),
$$

which implies 


$$
v_{r}(x) \geq R_{1}(x)+\frac{\rho R_{1}(0)}{1-\rho} .
$$

If $r>\frac{\rho R_{1}(1)}{1-\rho}$, it follows from (D.7) that

$$
\rho \int_{0}^{1} v_{r}(y) Q_{0}(d y \mid x)<\rho v_{r}(0)+r, \quad \forall x,
$$

i.e., $\theta(r)=1^{+}$.

Lemma D.2. There exists $\delta>0$ such that for all $0<r<\delta$,

$$
\rho \int_{0}^{1} v_{r}(y) Q_{0}(d y \mid x)>\rho v_{r}(0)+r, \quad \forall x,
$$

and so $\theta(r)=0$.

Proof. By (D.8),

$$
\begin{aligned}
\rho \int_{0}^{1} v_{r}(y) Q_{0}(d y \mid x) & \geq \rho \int_{0}^{1} R_{1}(y) Q_{0}(d y \mid x)+\frac{\rho^{2} R_{1}(0)}{1-\rho} \\
& \geq \rho \int_{0}^{1} R_{1}(y) Q_{0}(d y \mid 0)+\frac{\rho^{2} R_{1}(0)}{1-\rho},
\end{aligned}
$$

and (D.7) gives

$$
\rho v_{r}(0)+r \leq \frac{\rho R_{1}(0)}{1-\rho}+\frac{r}{1-\rho} .
$$

Since $R_{1}(x)$ is strictly increasing,

$$
C_{R_{1}}:=\int_{0}^{1} R_{1}(y) Q_{0}(d y \mid 0)-R_{1}(0)>0 .
$$

And we have

$$
\rho \int_{0}^{1} v_{r}(y) Q_{0}(d y \mid x)-\left(\rho v_{r}(0)+r\right) \geq \rho C_{R_{1}}-\frac{r}{1-\rho} .
$$

It suffices to take $\delta=\rho(1-\rho) C_{R_{1}}$.

Define the nonempty sets

$$
\mathscr{R}_{a_{0}}=\{r>0 \mid(\overline{D .9}) \text { hods }\}, \quad \mathscr{R}_{a_{1}}=\{r>0 \mid(\overline{D .10}) \text { holds }\} .
$$

Remark D.1. We have $\left(\frac{\rho R_{1}(1)}{1-\rho}, \infty\right) \subset \mathscr{R}_{a_{0}}$ and $(0, \delta) \subset \mathscr{R}_{a_{1}}$.

Lemma D.3. Let $\left(r, v_{r}\right)$ be the parameter and the associated solution in (D.6). i) If $r>0$ satisfies 


$$
\rho \int_{0}^{1} v_{r}(y) Q_{0}(d y \mid x) \leq \rho v_{r}(0)+r, \quad \forall x,
$$

then any $r^{\prime}>r$ is in $\mathscr{R}_{a_{0}}$.

ii) If $r>0$ satisfies

$$
\rho \int_{0}^{1} v_{r}(y) Q_{0}(d y \mid x) \geq \rho v_{r}(0)+r, \quad \forall x,
$$

then any $r^{\prime} \in(0, r)$ is in $\mathscr{R}_{a_{1}}$.

Proof. i) For $r^{\prime}>r, v_{r^{\prime}}$ is uniquely solved from (D.6) with $r^{\prime}$ in place of $r$. We can use (D.11) to verify

$$
v_{r}(x)=\min \left[\rho \int_{0}^{1} v_{r}(y) Q_{0}(d y \mid x)+R_{1}(x), \quad \rho v_{r}(0)+R_{1}(x)+r^{\prime}\right] .
$$

Hence $v_{r^{\prime}}=v_{r}$ for all $x \in[0,1]$. It follows that $\rho \int_{0}^{1} v_{r^{\prime}}(y) Q_{0}(d y \mid x)<\rho v_{r^{\prime}}(0)+r^{\prime}$ for all $x$. Hence $r^{\prime} \in \mathscr{R}_{a_{0}}$.

ii) By (D.6) and (D.12), $v_{r}(0)=\frac{R_{1}(0)+r}{1-\rho}$, and subsequently,

$$
v_{r}(x)=\rho v_{r}(0)+R_{1}(x)+r=\frac{\rho R_{1}(0)+r}{1-\rho}+R_{1}(x) .
$$

By substituting $v_{r}(0)$ and $v_{r}(x)$ into $(\overline{\mathrm{D} .12})$, we obtain

$$
\rho R_{1}(0)+r \leq \rho \int_{0}^{1} R_{1}(y) Q_{0}(d y \mid x), \quad \forall x .
$$

Now for $0<r^{\prime}<r$, we construct $v_{r^{\prime}}(x)$, as a candidate solution to (D.6) with $r$ replaced by $r^{\prime}$, to satisfy

$$
v_{r^{\prime}}(0)=\rho v_{r^{\prime}}(0)+R_{1}(0)+r^{\prime}, \quad v_{r^{\prime}}(x)=\rho v_{r^{\prime}}(0)+R_{1}(x)+r^{\prime},
$$

which gives

$$
v_{r^{\prime}}(x)=\frac{\rho R_{1}(0)+r^{\prime}}{1-\rho}+R_{1}(x) .
$$

We show that $v_{r^{\prime}}(x)$ in (D.15) satisfies

$$
\rho v_{r^{\prime}}(0)+r^{\prime}<\rho \int_{0}^{1} v_{r^{\prime}}(y) Q_{0}(d y \mid x), \quad \forall x,
$$

which is equivalent to $\rho R_{1}(0)+r^{\prime}<\rho \int_{0}^{1} R_{1}(y) Q_{0}(d y \mid x)$ for all $x$, which in turn follows from (D.13). By (D.14) and (D.16), $v_{r^{\prime}}$ indeed satisfies (D.6) with $r$ replaced by $r^{\prime}$. So $r^{\prime} \in \mathscr{R}_{a_{1}}$.

Further define 


$$
\underline{r}=\sup \mathscr{R}_{a_{1}}, \quad \bar{r}=\inf \mathscr{R}_{a_{0}} .
$$

Lemma D.4. $i) \underline{r}$ satisfies $\rho \int_{0}^{1} v_{\underline{r}}(y) Q_{0}(d y \mid 0)=\rho v_{\underline{r}}(0)+\underline{r}$, and $\theta(\underline{r})=0$.

ii) $\bar{r}$ satisfies $\rho \int_{0}^{1} v_{\bar{r}}(y) Q_{0}(d y \mid 1)=\rho v_{\bar{r}}(1)=\rho v_{\bar{r}}(0)+\bar{r}$, and $\theta(\bar{r})=1$.

iii) We have $0<\underline{r}<\bar{r}<\infty$.

iv) The threshold $\theta(r)$ as a function of $r \in(0, \infty)$ is continuous and strictly increasing on $[\underline{r}, \bar{r}]$.

Proof. i)-ii) By Lemmas D.2 and D.3 we have $0<\underline{r} \leq \infty$ and $0 \leq \bar{r}<\infty$. Assume $\underline{r}=\infty$; then $\mathscr{R}_{a_{1}}=(0, \infty)$ giving $\mathscr{R}_{a_{0}}=\emptyset$, a contradiction. So $0<\underline{r}<\infty$. For $\delta>0$ in Lemma D.2 we have $(0, \delta) \subset \mathscr{R}_{a_{1}}$. Therefore, $0<\bar{r}<\infty$. Note that $v_{r}$ depends on the parameter $r$ continuously, i.e., $\lim _{\left|r^{\prime}-r\right| \rightarrow 0} \sup _{x}\left|v_{r^{\prime}}(x)-v_{r}(x)\right|=0$. Hence

$$
\rho \int_{0}^{1} v_{\underline{r}}(y) Q_{0}(d y \mid 0) \geq \rho v_{\underline{r}}(0)+\underline{r} .
$$

Now assume

$$
\rho \int_{0}^{1} v_{\underline{r}}(y) Q_{0}(d y \mid 0)>\rho v_{\underline{r}}(0)+\underline{r} .
$$

Then there exists a sufficiently small $\varepsilon>0$ such that $(\mathrm{D}$.17) still holds when $(\underline{r}+$ $\left.\varepsilon, v_{\underline{r}+\varepsilon}\right)$ replaces $\left(\underline{r}, v_{\underline{\underline{r}}}\right)$; since $g(x)=\int_{0}^{1} v_{\underline{\underline{x}}+\varepsilon}(y) Q_{0}(d y \mid x)$ is increasing in $x$, then $\underline{r}+\varepsilon \in \mathscr{R}_{a_{1}}$, which is impossible. Hence (D.17) does not hold, and this proves i). ii) can be shown in a similar manner.

To show iii), assume

$$
0<\bar{r}<\underline{r}<\infty .
$$

Then, recalling Remark D.1, there exist $r^{\prime} \in \mathscr{R}_{a_{0}}$ and $r^{\prime \prime} \in \mathscr{R}_{a_{1}}$ such that

$$
0<\bar{r}<r^{\prime}<r^{\prime \prime}<\underline{r}<\infty .
$$

By Lemma D.3-i), $r^{\prime \prime} \in \mathscr{R}_{a_{0}}$, and then $r^{\prime \prime} \in \mathscr{R}_{a_{0}} \cap \mathscr{R}_{a_{1}}=\emptyset$, which is impossible. Therefore, (D.18) does not hold and we conclude $0<\underline{r} \leq \bar{r}<\infty$. We further assume $\underline{r}=\bar{r}$. Then i)-ii) would imply $\int_{0}^{1} v_{\underline{r}}(y) Q_{0}(d y \mid 0)=v_{\underline{r}}(1)$, which is impossible since $v_{\underline{r}}$ is strictly increasing on $[0,1]$ and (A3) holds. This proves iii).

iv) By the definition of $\underline{r}$ and $\bar{r}$, it can be shown using (D.6) that $\theta(r) \in(0,1)$ for $r \in(\underline{r}, \bar{r})$. By the continuous dependence of the function $v_{r}(\cdot)$ on $r$ and the method of proving [27, Lemma 10], we can show the continuity of $\theta(r)$ on $(0,1)$, and further show $\lim _{r \rightarrow \underline{r}^{+}} \theta(r)=0$ and $\lim _{r \rightarrow \bar{r}^{-}} \theta(r)=1$. So $\theta(r)$ is continuous on $[\underline{r}, \bar{r}]$. If $\theta(r)$ were not strictly increasing on $[\underline{r}, \bar{r}]$, there would exist $\underline{r}<r_{1}<r_{2}<\bar{r}$ such that

$$
\theta\left(r_{1}\right) \geq \theta\left(r_{2}\right) .
$$

If $\theta\left(r_{1}\right)>\theta\left(r_{2}\right)$ in (D.19), by the continuity of $\theta(r), \theta(\underline{r})=0, \theta(\bar{r})=1$, and the intermediate value theorem we may find $r^{\prime} \in\left(\underline{r}, r_{1}\right)$ such that $\theta\left(r_{1}^{\prime}\right)=\theta\left(r_{2}\right)$. Next, we replace $r_{1}$ by $r_{1}^{\prime}$. Thus if $\theta(r)$ is not strictly increasing, we may find $r_{1}<r_{2}$ from 
$(\underline{r}, \bar{r})$ such that $\theta\left(r_{1}\right)=\theta\left(r_{2}\right) \in(0,1)$, which is a contradiction to LemmaD.1 This proves iv).

Remark D.2. By Lemmas D.3 and D.4, $\mathscr{R}_{a_{1}}=(0, \underline{r})$ and $\mathscr{R}_{a_{0}}=(\bar{r}, \infty)$.

\section{Acknowledgement}

We would like to thank Aditya Mahajan for helpful discussions.

\section{References}

1. Acemoglu, D., Jensen, M.K.: Aggregate comparative statics. Games and Economic Behavior 81, 27-49 (2013)

2. Acemoglu, D., Jensen, M.K.: Robust comparative statics in large dynamic economies. Journal of Political Economy 123, 587-640 (2015)

3. Adlakha, S., Johari, R., Weintraub, G.Y.: Equilibria of dynamic games with many players: Existence, approximation, and market structure. J. Econ. Theory 156, 269-316 (2015)

4. Altman, E., Stidham, S.: Optimality of monotonic policies for two-action Markovian decision processes, with applications to control of queues with delayed information. Queueing Systems 21, 267-291 (1995)

5. Amir R.: Sensitivity analysis of multisector optimal economic dynamics. Journal of Mathematical Economics 25, 123-141 (1996)

6. Asmussen, S.: Applied Probability and Queues, 2nd edn. Springer, New York (2003)

7. Athreya, K.B., Roy, V.: When is a Markov chain regenerative? Statistics and Probability Letters 84, 22-26 (2014)

8. Babichenko, Y.: Best-reply dynamics in large binary-choice anonymous games. Games and Economic Behavior 81, 130-144 (2013)

9. Bardi, M.: Explicit solutions of some linear-quadratic mean field games. Netw. Heterogeneous Media 7, 243-261 (2012)

10. Bauerle, N., Rieder, U.: Markov Decision Processes with Applications to Finance. Springer, Berlin (2011)

11. Becker R. A.: Comparative dynamics in aggregate models of optimal capital accumulation. Quarterly Journal of Economics 100, 1235-1256 (1985)

12. Bensoussan, A., Frehse, J., Yam, P.: Mean Field Games and Mean Field Type Control Theory. Springer, New York (2013)

13. Biswas, A.: Mean field games with ergodic cost for discrete time Markov processes, preprint, arXiv: $1510.08968,2015$.

14. Bonnans, J.F., Shapiro, A.: Perturbation Analysis of Optimization Problems. Springer-Verlag, New York (2000)

15. Brock, W.A., Durlauf, S. N.: Discrete choice with social interactions. Rev. Econ. Studies 68, 235-260 (2001)

16. Caines, P.E.: Mean field games. In: Samad, T., Baillieul, J. (eds.) Encyclopedia of Systems and Control. Springer-Verlag, Berlin (2014)

17. Caines, P.E., Huang, M., Malhamé, R.P.: Mean Field Games. In: Basar, T., Zaccour, G. (eds.) Handbook of Dynamic Game Theory, pp. 345-372, Springer, Berlin (2017)

18. Cardaliaguet, P.: Notes on mean field games, University of Paris, Dauphine (2012)

19. Carmona R., Delarue, F.: Probabilistic Theory of Mean Field Games with Applications, vol I and II. Springer, Cham (2018) 
20. Dorato, P.: On sensitivity in optimal control systems. IEEE Transactions on Automatic Control 8, 256-257 (1963)

21. Filar, J.A., Vrieze, K.: Competitive Markov Decision Processes. Springer, New York (1997)

22. Gomes, D. A., Mohr, J., Souza, R.R.: Discrete time, finite state space mean field games. J. Math. Pures Appl. 93 308-328, (2010)

23. Hernandez-Lerma, O.: Adaptive Markov Control Processes. Springer-Verlag, New York (1989)

24. Hicks, J. R.: Value and Capital. Clarendon Press, Oxford (1939)

25. Huang, M., Caines, P.E., Malhamé, R.P.: Individual and mass behaviour in large population stochastic wireless power control problems: Centralized and Nash equilibrium solutions. Proc. 42nd IEEE Conference on Decision and Control, pp. 98-105, Maui, HI (2003)

26. Huang, M., Caines, P.E., Malhamé, R.P.: Large-population cost-coupled LQG problems with nonuniform agents: Individual-mass behavior and decentralized $\varepsilon$-Nash equilibria. IEEE Trans. Autom. Control 52, 1560-1571 (2007)

27. Huang, M., Ma, Y.: Mean field stochastic games: Monotone costs and threshold policies (in Chinese), Sci. Sin. Math. (special issue in honour of the 80th birthday of Prof. H-F. Chen) 46, 1445-1460 (2016)

28. Huang, M., Ma, Y.: Mean field stochastic games with binary action spaces and monotone costs. arXiv:1701.06661v1, 2017.

29. Huang, M., Ma, Y.: Mean field stochastic games with binary actions: Stationary threshold policies. Proc. 56th IEEE Conference on Decision and Control, Melbourne, Australia, pp. 27-32 (2017)

30. Huang, M., Malhamé, R.P., Caines, P.E.: Large population stochastic dynamic games: Closedloop McKean-Vlasov systems and the Nash certainty equivalence principle. Commun. Inform. Systems 6, 221-251 (2006)

31. Huang, M., Zhou, M..: Linear quadratic mean field games: Asymptotic solvability and relation to the fixed point approach. IEEE Transactions on Automatic Control (2018, in revision, conditionally accepted)

32. Ito, K., Kunisch, K.: Sensitivity analysis of solutions to optimization problems in Hilbert spaces with applications to optimal control and estimation. J. Differential Equations 99, 1-40 (1992)

33. Jovanovic, B., Rosenthal, R.W.: Anonymous sequential games. Journal of Mathematical Economics 17, 77-87 (1988)

34. Jiang, L., Anantharam, V., Walrand, J.: How bad are selfish investments in network security? IEEE/ACM Trans. Networking 19, 549-560 (2011)

35. Kolokoltsov, V.N.: Nonlinear Markov games on a finite state space (mean-field and binary interactions). International J. Statistics Probability 1, 77-91 (2012)

36. Kress, R.: Linear Integral Equations. Springer, Berlin (1989)

37. Lasry, J.-M., Lions, P.-L.: Mean field games. Japan. J. Math. 2, 229-260 (2007)

38. Lelarge, M., Bolot, J.: A local mean field analysis of security investments in networks. Proc. ACM SIGCOMM NetEcon, Seattle, WA, pp. 25-30, 2008

39. Li, T., Zhang, J.-F.: Asymptotically optimal decentralized control for large population stochastic multiagent systems. IEEE Trans. Autom. Control 53, 1643-1660 (2008)

40. Manfredia, P., Posta, P.D., dÓnofrio, A., Salinelli, E., Centrone, F., Meo, C., Poletti, P.: Optimal vaccination choice, vaccination games, and rational exemption: An appraisal. Vaccine 28, 98-109 (2010)

41. Meyn, S., Tweedie, R. L.: Markov Chains and Stochastic Stability, 2nd ed. Cambridge University Press, Cambridge (2009)

42. Milgrom, P., Shannon, C.: Monotone comparative statics. Econometrica 62, 157-80 (1994)

43. Moon, J., Basar, T.: Linear quadratic risk-sensitive and robust mean field games. IEEE Trans. Autom. Control 62, 1062-1077 (2017)

44. Müller, A., Stoyan, D.: Comparison Methods for Stochastic Models and Risks. Wiley, Chichester (2002)

45. Oniki, H.: Comparative dynamics (sensitivity analysis) in optimal control theory. J. Econ. Theory 6, 265-283 (1973) 
46. Saldi, N., Basar, T., Raginsky, M.: Markov-Nash equilibria in mean-field games with discounted cost. SIAM J. Control Optimization 56, 4256-4287 (2018)

47. Samuelson, P.A.: Foundations of Economic Analysis, enlarged edn., Harvard University Press, Cambridge, MA (1983)

48. Schelling, T.C.: Hockey helmets, concealed weapons, and daylight saving: A study of binary choices with externalities. The Journal of Conflict Resolution 17, 381-428 (1973)

49. Selten, R.: An axiomatic theory of a risk dominance measure for bipolar games with linear incentives. Games and Econ. Behav. 8, 213-263 (1995)

50. Shapley, L.S.: Stochastic games. Proc. Natl. Acad. Sci. 39, 1095-1100 (1953)

51. Sigman, K., Wolff, R.W.: A review of regenerative processes. SIAM Rev. 35, 269-288 (1993)

52. Sun, Y.: The exact law of large numbers via Fubini extension and characterization of insurable risks. J. Econ. Theory 126, 31-69 (2006)

53. Topkis, D.M.: Supermodularity and Complementarity. Princeton Univ. Press, Princeton (1998)

54. Walker, M., Wooders, J., Amir, R.: Equilibrium play in matches: Binary Markov games. Games and Economic Behavior 71, 487-502 (2011)

55. Weintraub, G.Y., Benkard, C.L., Van Roy, B.: Markov perfect industry dynamics with many firms. Econometrica 76, 1375-1411 (2008)

56. Yong, J.: Linear-quadratic optimal control problems for mean-field stochastic differential equations. SIAM J. Control Optim. 51, 2809-2838 (2013)

57. Zhou, M., Huang, M.: Mean field games with Poisson point processes and impulse control. Proc. 56th IEEE Conference on Decision and Control, Melbourne, Australia pp. 3152-3157 (2017) 